\title{
Orbital-resolved vortex-core states in FeSe superconductors: A calculation based on a three-orbital model
}

\author{
Q. E. Wang ${ }^{1}$ and F. C. Zhang ${ }^{1,2,3, *}$ \\ ${ }^{1}$ Department of Physics and Center of Theoretical and Computational Physics, The University of Hong Kong, Hong Kong, China \\ ${ }^{2}$ Department of Physics, Zhejiang University, Hangzhou, China \\ ${ }^{3}$ Collaborative Innovation Center of Advanced Microstructures, Nanjing 210093, China \\ (Received 8 April 2014; revised manuscript received 26 May 2015; published 15 June 2015)
}

\begin{abstract}
We study the electronic structure of vortex core states of FeSe superconductors based on a $t_{2 g}$ three-orbital model by solving the Bogoliubov-de Gennes (BdG) equation self-consistently. The orbital-resolved vortex core states of different pairing symmetries manifest themselves as distinguishable structures due to different quasiparticle wave functions. The obtained vortices are classified in terms of the invariant subgroups of the symmetry group of the mean-field Hamiltonian in the presence of a magnetic field. Isotropic $s$ and anisotropic $s$-wave vortices have $G_{5}$ symmetry for each orbital, whereas $d_{x^{2}-y^{2}}$-wave vortices show $G_{6}^{*}$ symmetry for $d_{x z / y z}$ orbitals and $G_{5}^{*}$ symmetry for $d_{x y}$ orbital. In the case of $d_{x^{2}-y^{2}}$-wave vortices, hybridized-pairing between $d_{x z}$ and $d_{y z}$ orbitals gives rise to a relative phase difference in terms of gauge transformed pairing order parameters between $d_{x z / y z}$ and $d_{x y}$ orbitals, which is essentially caused by a transformation of co-representation of $G_{5}^{*}$ and $G_{6}^{*}$ subgroups. The calculated local density of states (LDOS) of $d_{x^{2}-y^{2}}$-wave vortices shows a qualitatively similar pattern with the experimental results. The phase difference of $\frac{\pi}{4}$ between $d_{x z / y z}$ and $d_{x y}$ orbital-resolved $d_{x^{2}-y^{2}}$-wave vortices can be verified by further experimental observation.
\end{abstract}

DOI: 10.1103/PhysRevB.91.214509

PACS number(s): 74.70.Xa, 74.25.Wx, 74.20.-z

\section{INTRODUCTION}

Quantized vortices, as stable topological defects, observed in a variety of quantum systems such as superconductors and superfluids, are characterized by their nature of soliton solutions of dynamical systems [1]. The electronic structure of vortices in cuprate superconductors exhibits charging effects [2-4] and is anisotropic due to the $d_{x^{2}-y^{2}}$-wave pairing symmetry [5]. Earlier theoretical works have investigated the vortex line states based on microscopic models [6-9]. In iron-based superconductors, the band structure and multiorbital pairings play an important role and the vortex structure may be richer due to the multiorbital dependency. Vortex core states of two-fold rotational symmetry, which are attributed to the orbital-dependent reconstruction in FeSe superconductors [10], have been reported by Song et al. from a scanning tunneling microscopy (STM) experiment [11].

In most of iron-based superconductors, the Fermi surfaces consist of both electron pockets around the $M$ point at the corners and hole pockets around the $\Gamma$ point of the folded Brilliouin zone (BZ). An $s_{ \pm}$-wave superconducting (SC) pairing symmetry has been proposed, where the pairing order parameters at the electron and hole pockets have opposite signs [13-22]. FeSe superconductor is interesting for its unique electronic structure in which only electron pockets are found and the hole pockets are well below the Fermi level, as angleresolved photoemission spectroscopy (ARPES) shows [12]. When the hole pocket at $\Gamma$ vanishes, we do not have an $s_{ \pm}$-wave pairing state anymore and instead $s$ and $d_{x^{2}-y^{2}}$-wave pairing states should be considered $[23,24]$. The iron-based superconductivity without a hole pocket is a great challenge to the weak-coupling theory where the superconductivity is proposed to be driven by the nesting of the electron and hole

*Corresponding author: fuchun@hku.hk
Fermi surfaces. The absence of the hole pocket in FeSe makes the argument difficult.

Vortex structures in iron-based superconductors have been studied by a number of authors [25-29]. These studies are mainly based on band structures having a hole pocket at $\Gamma$. We expect the vortex structure be affected by the Fermi surface topology. In this work, we use a three-orbital model to study the vortex structure of the FeSe SC state. The three-orbital microscopic model reproduces qualitatively the correct Fermi surface with only electron pockets. We solve the BdG equation self-consistently to study the orbitalresolved vortex core states for various SC pairing symmetries: isotropic $s$ (on-site pairing), anisotropic $s$ (nextnearest-neighbor site pairing), and $d_{x^{2}-y^{2}}$ (nearest-neighbor site pairing) waves. We compare the results of our calculations with those observed in a recent STM experiment on an FeSe vortex and suggest that the pairing symmetry is a $d_{x^{2}-y^{2}}$ wave. We predict that there is a relative phase difference about $\frac{\pi}{4}$ between the pairing order parameters defined on $d_{x z / y z}$ and $d_{x y}$ orbitals in the case of $d_{x^{2}-y^{2}}$ orbital-resolved vortices, while such a phase difference is trivial in the case of isotropic $s$ and anisotropic $s$-wave vortices. The paper is organized as follows. An introduction of the magnetic translation group and the classification of vortex solutions are given in Sec. II. In Sec. III, we present the three-orbital model and the selfconsistent BdG approach. In Sec. IV, we discuss the properties of the vortex core states for different pairing symmetries and compare our results with experimental observations. Finally, a summary is given in Sec. V.

\section{MAGNETIC TRANSLATIONAL SYMMETRY AND WINDING STRUCTURES OF A SINGLE VORTEX}

From a theoretical point of view, a vortex lattice in the mixed states of type II superconductors is the ground state 
of a fermionic system which is characterized by interaction between a homogenous magnetic field with $C_{\infty}$ symmetry and Cooper pairs with a definite SC pairing symmetry [30]. In ironbased superconductors, the situation becomes complicated because of orbital degrees of freedom. Consequently, the crystal symmetry, band structure, and SC pairing symmetry, determine the electronic structure of vortices. Among these constraints of symmetry, the vortex structures are mainly dominated by magnetic translation invariance, whose generator are the crystal momentum and the vector potential of a magnetic field [31]. However, such a conventional magnetic translation group defines a magnetic unit cell containing two vortices. It is not the symmetry group of Abrikosov lattice in which only a single vortex is stabilized within one magnetic unit cell. A breakthrough in relation to this difficulty was presented by Ozaki et al. [32]. In their work, the magnetic translation group describing a single vortex was discovered to be a subgroup of the direct product of a conventional magnetic translation group and a gauge transformation group U(1). Therefore a stable vortex structure can be solved numerically in one magnetic unit cell by taking advantage of the nontrivial winding boundary conditions derived from the properties of the magnetic translation group [33].

Instead of doing calculations of two vortices in one magnetic unit cell, we follow the method given by Ozaki et al. [32,33], in which only single vortex structures are calculated in one magnetic unit cell, so that the calculated results can be classified by irreducible representations of the magnetic translation group. The numerical calculations in previous works, as mentioned above [25-27], are mostly carried out for two vortices in one magnetic unit cell. These vortex states, however, can not be identified by invariant subgroups of the magnetic translation group because they belong to the irreducible representations of the conventional magnetic translation group. Furthermore, two vortices in one magnetic unit cell are not independent because the induction of interaction between them. It is well known that the topological defects in unconventional superconductors and superfluids with certain symmetry breaking behave distinguishably from the conventional singular (hard core) vortices [34]. For instance, a vortex in ${ }^{3} \mathrm{He}$ has a finite amplitude of order parameters in the soft core region whose size is larger than the coherent length, whereas the winding structure is nontrivial. Therefore, in our numerical calculation, we concentrate on the winding structures of vortices for each orbital, although the vortices in iron-based superconductors are mostly a hard core feature, and classify the vortex structures of isotropic $s$-, anisotropic $s$-, and $d_{x^{2}-y^{2}}$-wave pairing symmetries in terms of invariant subgroups of the magnetic translation group. Special attention will be paid to the difference of vortex states defined between $A_{1 g}$ (isotropic $s$ and anisotropic $s$ wave) and $B_{1 g}\left(d_{x^{2}-y^{2}}\right.$ wave $)$ irreducible unitary representations of $D_{4}$ group.

The Hamiltonian of the SC system in the presence of a homogeneous magnetic field along the $\hat{z}$ direction is obtained from its zero-field form by modifying the hopping and pairing terms with a Peierls phase [35], respectively, which is of the following form:

$$
\begin{aligned}
H & =H_{0}+H_{\text {pair }}, \\
H_{0} & =\sum_{i, j, \alpha, \beta, \sigma}\left[\tilde{t}_{\sigma \sigma}(i \alpha, j \beta)-\mu \delta_{i j} \delta_{\alpha \beta}\right] a_{i \alpha \sigma}^{\dagger} a_{j \beta \sigma}, \\
H_{\text {pair }} & =\sum_{i, j, \alpha, \beta}\left[\tilde{\Delta}_{\uparrow \downarrow}(i \alpha, j \beta) a_{i \alpha \uparrow}^{\dagger} a_{j \beta \downarrow}^{\dagger}+\text { H.c. }\right],
\end{aligned}
$$

in which

$$
\begin{aligned}
& \tilde{t}_{\sigma \sigma}(i \alpha, j \beta)=t_{\sigma \sigma}(i \alpha, j \beta) \exp \left[\frac{i e}{\hbar c} \int_{j}^{i} \vec{A}(\vec{r}) \cdot d \vec{r}\right], \\
& \tilde{\Delta}_{\uparrow \downarrow}(i \alpha, j \beta)=\Delta_{\uparrow \downarrow}(i \alpha, j \beta) \exp [i \phi(i, j)],
\end{aligned}
$$

where $a_{i \alpha \sigma}^{\dagger}\left(a_{i \alpha \sigma}\right)$ denotes the creation(annihilation) operator of electrons with spin $\sigma=\uparrow, \downarrow$ and orbital $\alpha$ at site $i$. $t_{\sigma \sigma}(i \alpha, j \beta)$ are hopping integrals and $\mu$ is the chemical potential. We assume that the screening magnetic field inside the superconductor can be neglected except for that the magnetic field is close to the upper critical field. The SC pairing mechanism has been proposed to be of magnetic origin. In this paper, however, we shall focus on the vortex core state and start from an extended attractive Hubbard model for simplicity. The SC order parameter stemming from the mean-field decoupling of the paired scattering term is expressed as $\Delta_{\uparrow \downarrow}(i \alpha, j \beta)=V_{\uparrow \downarrow}(i \alpha, j \beta)\left\langle a_{j \beta \downarrow} a_{i \alpha \uparrow}\right\rangle$ for a singlet pairing channel. The Peierls phase [35] in hopping terms comes from the fact that the Lagrangian of electron in a magnetic field contains a dynamical term $\frac{e}{c} \vec{v} \cdot \vec{A}$, which gives rise to the phase accumulation in the propagator of an electron describing the hopping process between two lattice sites. The modification of pairing order parameters accounts for eliminating the mixing of different pairing states under the action of the magnetic translation group. The mathematical interpretation of doing this is essentially searching for gauge transformed order parameters, which span a representation of magnetic translation group $[32,33]$. The gauge transformation, carried out by phase $\phi(i, j)$, has different definition with respect to anisotropic $s$ - and $d_{x^{2}-y^{2}}$-wave pairing states, whereas in the case of isotropic $s$-wave pairing it is trivial. The gauge transformed order parameter for $d_{x^{2}-y^{2}}$-wave pairing has been derived by means of a group theoretical analysis [32,33]. The magnetic translation operator takes the following form in a symmetric gauge $\vec{A}=-\frac{1}{2} \vec{r} \times \vec{B}$, when it acts on creation operators [31,32]:

$$
\begin{aligned}
L\left(\vec{R}_{\lambda}\right) a_{i \alpha \sigma}^{\dagger} & =e^{i \frac{\pi}{2}\left(\mathcal{N}_{v} \lambda_{x} \lambda_{y}\right)} T\left(\vec{R}_{\lambda}\right) a_{i \alpha \sigma}^{\dagger} \\
& =e^{i \frac{\pi}{2} \mathcal{N}_{v}\left[\lambda_{x} \lambda_{y}+\frac{1}{N}\left(\lambda_{x} i_{y}-\lambda_{y} i_{x}\right)\right]} a_{i+\lambda, \alpha \sigma}^{\dagger},
\end{aligned}
$$

and the resultant transformation of order parameter is

$$
\begin{aligned}
& \left\langle a_{j+\lambda, \beta \downarrow} a_{i+\lambda, \alpha \uparrow}\right\rangle \\
& =e^{i \pi \mathcal{N}_{v}\left[\lambda_{x} \lambda_{y}+\frac{1}{2 N} \lambda_{x}\left(i_{y}+j_{y}\right)-\frac{1}{2 N} \lambda_{y}\left(i_{x}+j_{x}\right)\right]}\left\langle a_{j \beta \downarrow} a_{i \alpha \uparrow}\right\rangle,
\end{aligned}
$$

where $\vec{R}_{\lambda}=\lambda_{x} N \hat{x}+\lambda_{x} N \hat{y}$ is the basis vector of the magnetic unit cell containing $N$ lattice sites and $\mathcal{N}_{v}$ is the number of vortices within one magnetic unit cell. We have restricted ourselves to the cases of a square vortex lattice with lattice 
constant set to unity. Equation (3) defines the actions of the magnetic translation group $\left\{L\left(\vec{R}_{\lambda}\right)\right\}$ on the field operators, and all of the operations form a group in the representation space spanned by the gauge transformed order parameters, provided that a certain group condition is satisfied. Note that the gauge transformation, as an internal symmetry transformation, takes its complex conjugate form when acts on annihilation operators. Different from the situation for a conventional magnetic translation group [31] $\left\{T\left(\vec{R}_{\lambda}\right)\right\}: \mathcal{N}_{v}=2$, the group condition of a magnetic translation group, which is the symmetry group of Abrikosov lattice, is that only a single magnetic flux $\varphi_{0}=\frac{h c}{2 e}$ is contained in one magnetic unit cell [32,33], i.e., $\mathcal{N}_{v}=1$. It has been pointed out that $d_{x^{2}-y^{2}} \sim \cos \left(k_{x}\right)-\cos \left(k_{y}\right)$-wave order parameters will mix with extended $s^{*} \sim \cos \left(k_{x}\right)+\cos \left(k_{y}\right)$, $p_{x} \sim i \sin \left(k_{x}\right)$, and $p_{y} \sim i \sin \left(k_{y}\right)$-wave order parameters under the operation of the magnetic translation group [32,33]. Such a mixing originates from the fact that the symmetry group of the normal state Hamiltonian contains a local gauge transformation generated by the vector potential of a magnetic field. The redefined SC gauge transformed order parameters transforming according to invariant subgroups of $D_{4}$ group without any gauge component, as order parameters do in the absence of a magnetic field, are obtained by generating all of them with the action of a conjugate rotation subgroup $\left\{C_{4 z}^{k}\left(i_{x}, j_{y}\right), k=1,2,3,4\right\}$ on one of the pairing bonds of every local order parameter accompanied by a Peierls phase factor [35]. The generator of conjugate rotation subgroup is defined as

$$
C_{4 z}\left(i_{x}, j_{y}\right)=T\left(i_{x}, j_{y}\right) C_{4 z} T^{-1}\left(i_{x}, j_{y}\right),
$$

where $C_{4 z}$ is a fourfold rotation around the origin of the coordinate system. Therefore the mixing of order parameters under magnetic translation is eliminated by redefining the rotations of all local order parameters at different sites back

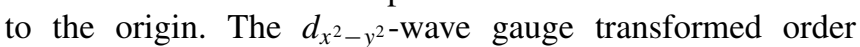
parameter is consequently redefined as

$$
\begin{aligned}
& \tilde{\Delta}_{\uparrow \downarrow}^{d_{x^{2}-y^{2}}}(i \alpha, j \beta) \\
& \quad=\frac{V_{\uparrow \downarrow}(j \beta, i \alpha)}{2}\left\langle a_{i \alpha \downarrow} a_{j \beta \uparrow}\right\rangle\left(e^{ \pm i K i_{y}} \delta_{i \pm \hat{x}, j}-e^{\mp i K i_{x}} \delta_{i \pm \hat{y}, j}\right),
\end{aligned}
$$

where $K=\frac{\pi \mathcal{N}_{v}}{2 N^{2}}$ and $\hat{x}(\hat{y})$ denote the unit vectors of the two-dimensional lattice. Note that for singlet pairing the order parameters are symmetric under exchange of site-orbital quantum numbers.

Here, we follow a method given by Ozaki et al. [32,33] to derive the gauge transformed order parameters for anisotropic $s \sim \cos \left(k_{x}\right) \cos \left(k_{y}\right)$-wave pairing symmetry. The results of action of the conjugate rotation subgroup on the pairing bond along the $\hat{x}+\hat{y}$ direction are

$$
\begin{aligned}
& C_{4 z}\left(i_{x}, i_{y}\right)\left\langle a_{i \alpha \downarrow} a_{i+\hat{x}+\hat{y}, \beta \uparrow}\right\rangle=e^{-2 i K i_{y}}\left\langle a_{i \alpha \downarrow} a_{i-\hat{x}+\hat{y}, \beta \uparrow}\right\rangle, \\
& C_{2 z}\left(i_{x}, i_{y}\right)\left\langle a_{i \alpha \downarrow} a_{i+\hat{x}+\hat{y}, \beta \uparrow}\right\rangle=e^{2 i K\left(i_{x}-i_{y}\right)}\left\langle a_{i \alpha \downarrow} a_{i-\hat{x}-\hat{y}, \beta \uparrow}\right\rangle, \\
& C_{4 z}^{3}\left(i_{x}, i_{y}\right)\left\langle a_{i \alpha \downarrow} a_{i+\hat{x}+\hat{y}, \beta \uparrow}\right\rangle=e^{2 i K i_{x}}\left\langle a_{i \alpha \downarrow} a_{i+\hat{x}-\hat{y}, \beta \uparrow}\right\rangle .
\end{aligned}
$$

Then, a symmetric phase rearrangement can be made by multiplying by a Peierls phase $e^{i K\left(i_{y}-i_{x}\right)}$ to regain the magnetic translational symmetry as following:

$$
\begin{aligned}
& \tilde{\Delta}_{\uparrow \downarrow}^{\text {anis. } s}(i \alpha, j \beta) \\
& =\frac{V_{\uparrow \downarrow}(j \beta, i \alpha)}{4}\left\langle a_{i \alpha \downarrow} a_{j \beta \uparrow}\right\rangle\left[e^{i K\left(i_{y}-i_{x}\right)} \delta_{i+\hat{x}+\hat{y}, j}\right. \\
& \quad+e^{-i K\left(i_{x}+i_{y}\right)} \delta_{i-\hat{x}+\hat{y}, j} \\
& \left.\quad+e^{-i K\left(i_{y}-i_{x}\right)} \delta_{i-\hat{x}-\hat{y}, j}+e^{i K\left(i_{x}+i_{y}\right)} \delta_{i+\hat{x}-\hat{y}, j}\right] .
\end{aligned}
$$

The magnetic translation property of gauge transformed order parameters for an anisotropic $s$-wave pairing state, which is consistent with a $d_{x^{2}-y^{2}}$ wave, is

$$
\begin{aligned}
& \tilde{\Delta}_{\uparrow \downarrow}^{\text {anis. } s}(i+\lambda, \alpha, j+\lambda, \beta) \\
& \quad=e^{i \pi \mathcal{N}_{v}\left[\lambda_{x} \lambda_{y}+\frac{1}{N}\left(\lambda_{x} i_{y}-\lambda_{y} i_{x}\right)\right]} \tilde{\Delta}_{\uparrow \downarrow}^{\text {anis. } s}(i \alpha, j \beta),
\end{aligned}
$$

where $j$ is always related to $i$ as the next-nearest-neighbor site pairing. Compare this expression with Eq. (4), it is obvious that the gauge transformed order parameters (referring to order parameters thereafter) now form a basis of representation of the magnetic translation group and the mixing between anisotropic $s$ - and $d_{x y}$-wave pairing states under the action of the magnetic translation group has been eliminated.

The SC ground states, in the absence of a magnetic field, can be classified by finding all the invariant subgroups of the symmetry group $D_{4} \otimes \mathrm{U}(1)$, which have a one-to-one correspondence to the irreducible unitary representation of the symmetry group of the normal state Hamiltonian [36,37]. In the case of $D_{4}$ point group symmetry, such a classification is obtained by the fact that $D_{4}$ has three invariant subgroups of index 2, and the two-dimensional cyclic group, as a subgroup of $\mathrm{U}(1)$, compensates the phase change of order parameters by $e^{i \pi}$ when the elements of coset representative act on them. In the same manner, the ground state of a vortex structure can also be classified by finding all the invariant subgroups of the symmetry group of the Hamiltonian in a magnetic field [32], and consequently the winding structure of the vortex core states has symmetry constraints of different classes. The topological characteristics of the vortex states are the location of the pinning center, the phase distribution of order parameters, and the winding number. It turns out that the winding number of vortices of different symmetry properties, having a structural vanishing region, can be calculated from the symmetry constraints of the corresponding maximal little groups. In the work of Ozaki et al. [32,33], the winding numbers $\mathcal{W}$ of $s^{*}$ - and $d_{x^{2}-y^{2}}$-wave vortices have been calculated. Here, we calculate $\mathcal{W}$ for anisotropic $s$ - and $d_{x y}$-wave states and list all the results in Table I, in which

$$
\begin{aligned}
& G^{l}=\left(e+t C_{2 x}\right) \tilde{C}^{l} \wedge L, \\
& \tilde{C}^{l}=\left\{e^{-\frac{\pi}{2} l k} C_{4 z}^{k}, k=1,2,3,4\right\} .
\end{aligned}
$$

Note that $\tilde{C}^{l}$ always acts on paired field operators rather than a single-particle operator. The derivation is based on the fact 
TABLE I. Winding number of order parameters for different pairing states. $G_{i}, i=1,2,3,4,5,6$ are six maximal little groups. $G_{5,6}^{*}$ differs from $G_{5,6}$ by taking the complex conjugate of gauge transformation. The order parameters transform according to the basis functions of $D_{4}$ group as $s$ wave: $\sim$ const., anisotropic $s$ wave: $\sim \cos \left(k_{x}\right) \cos \left(k_{y}\right)$, extended $s^{*}$ wave: $\sim \cos \left(k_{x}\right)+\cos \left(k_{y}\right)$, $d_{x^{2}-y^{2}}$ wave: $\sim \cos \left(k_{x}\right)-\cos \left(k_{y}\right)$, and $d_{x y}$ wave: $\sim \sin \left(k_{x}\right) \sin \left(k_{y}\right)$, respectively. The index $l$ is defined in Eq. (10).

\begin{tabular}{lrcc}
\hline \hline & $l$ & $\mathcal{W}\left(s\right.$, anis. $\left.s, s^{*}\right)$ & $\mathcal{W}\left(d_{x^{2}-y^{2}}, d_{x y}\right)$ \\
\hline$G_{1} \sim G_{2}$ & 0 & 0 & 2 or -2 \\
$G_{5} \sim G_{6}$ & 1 & 1 & 3 or -1 \\
$G_{5}^{*} \sim G_{6}^{*}$ & -1 & -1 & 1 or -3 \\
$G_{3} \sim G_{4}$ & 2 & 2 & 4 or 0 \\
\hline \hline
\end{tabular}

that the generator of $\tilde{C}^{l}$, as a symmetry transformation of order parameters, leaves them invariant [33]. The winding structures of $G_{5}^{*}$ and $G_{6}^{*}$ vortices, which have been obtained from our numerical calculations, are shown in Fig. 1, respectively, where they differ by a corepresentation transformation as

$$
G_{6}^{*}=\left(\frac{\widehat{3 \pi}}{4}\right)^{-1} G_{5}^{*} \frac{\widehat{3 \pi}}{4}, \quad G_{6}^{*}=\frac{\widehat{\pi}}{4} G_{5}^{*}\left(\frac{\widehat{\pi}}{4}\right)^{-1} .
$$

The gauge transformation of field operator is defined as $\widehat{\phi} a_{i \alpha \sigma}=e^{-i \frac{\phi}{2}} a_{i \alpha \sigma}$ [32]. Note that the global gauge transformations of $-\frac{3 \pi}{4}$ or $\frac{\pi}{4}$ are both allowed by group theory. But it turns out from our numerical calculation that the phase difference of $\frac{\pi}{4}$ is more energetically favorable.

\section{METHODOLOGY AND BAND MODEL}

It has been reported that the electronic structure of iron-based superconductors in the vicinity of the Fermi level is dominated by $d_{x z}, d_{y z}$, and $d_{x y}$ orbitals from a first-principles calculation [38], therefore it is feasible to calculate the vortex core states based on an effective three-

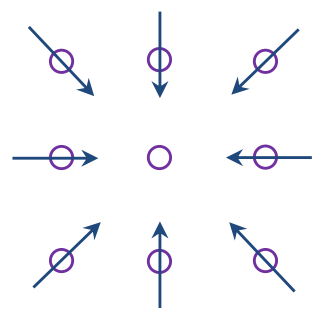

(a)

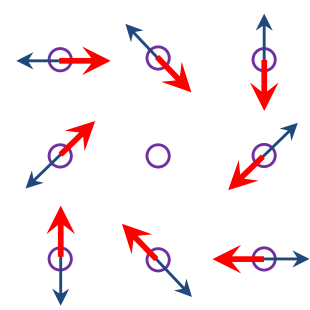

(b)
FIG. 1. (Color online) Schematic pictures showing the phase difference between $G_{5}^{*}$ (a) and $G_{6}^{*}$ (b) winding structures in the vicinity of the vortex core center [32]. The purple circles depict lattice sites on which the SC order parameters are defined and the arrows show the phase distribution of $\Delta_{\uparrow \downarrow}(i \alpha, j \beta)$. The blue arrows in (b) denote a phase difference of $-\frac{3 \pi}{4}$ and red arrows $\frac{\pi}{4}$ between $G_{6}^{*}$ and $G_{5}^{*}$ winding structures.

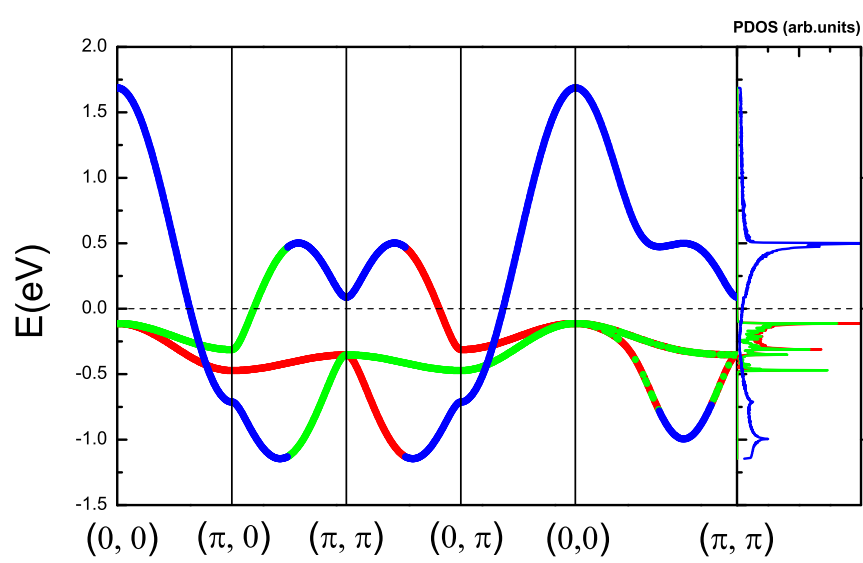

(a)

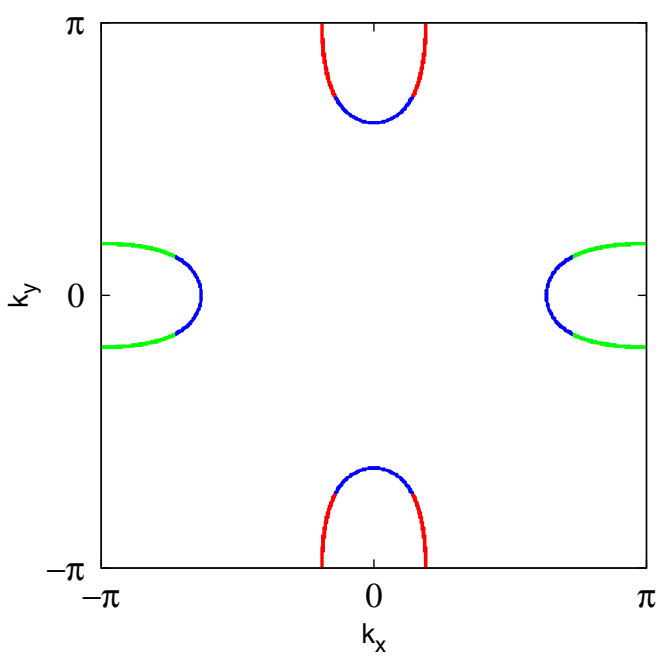

(b)

FIG. 2. (Color online) Orbital-resolved band structure, PDOS (a) and Fermi surface (b). The red $\left(d_{x z}\right)$, green $\left(d_{y z}\right)$, and blue $\left(d_{x y}\right)$ curves represent wight-dominating orbitals. The Fermi level has been set to zero.

orbital model [39]. Taking advantage of the fourfold rotational symmetry, the Blöch Hamiltonian can be written as

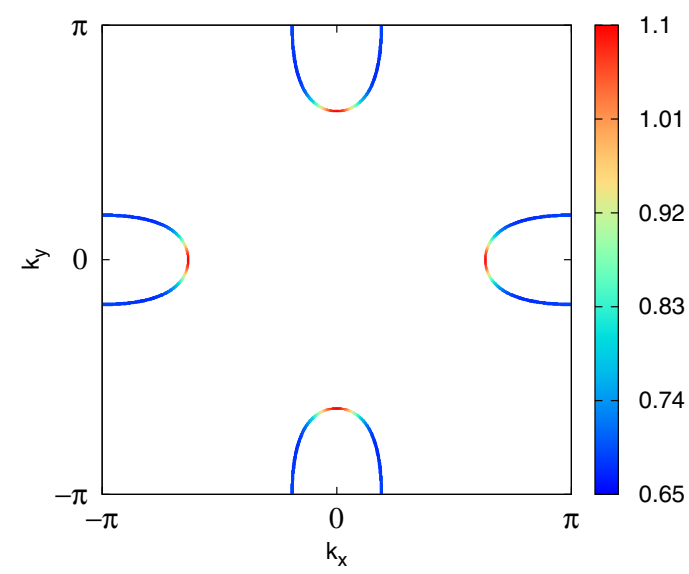

FIG. 3. (Color online) Color mapping of Fermi velocity $\hbar v_{F}$ (in unit $\mathrm{eV} \mathrm{m}$ ). 


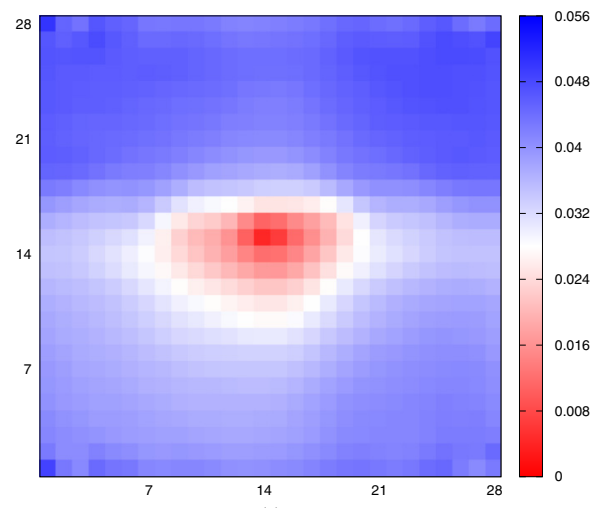

(a)

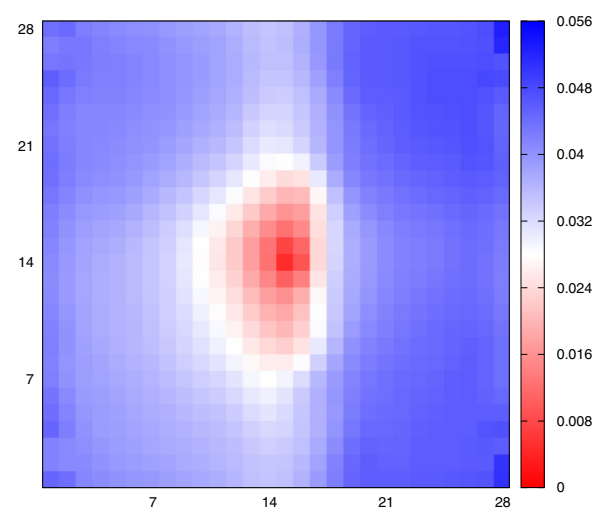

(c)

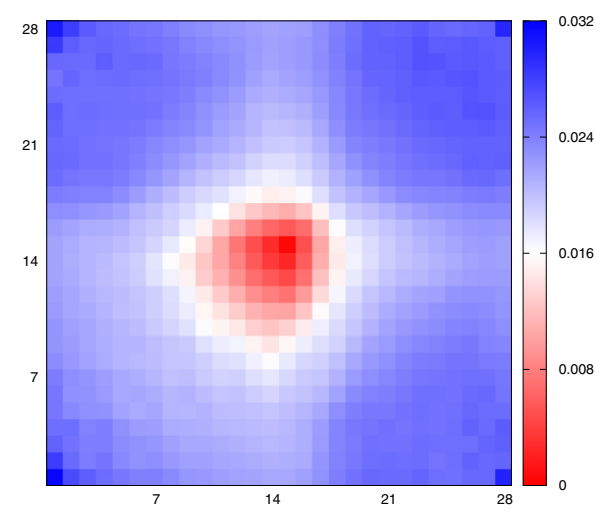

(e)

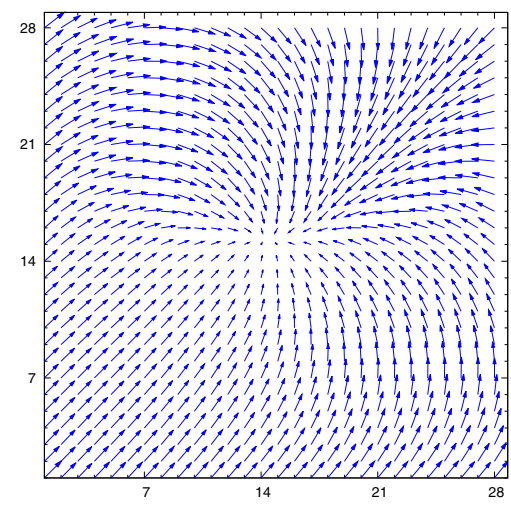

(b)

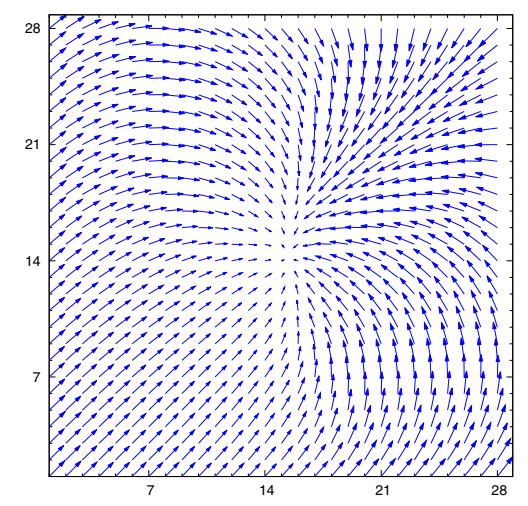

(d)

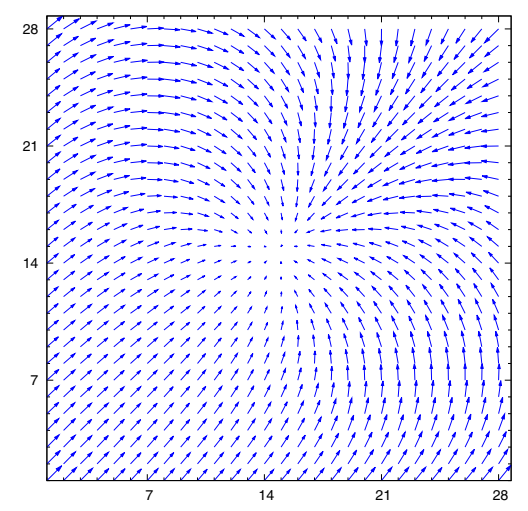

(f)

FIG. 4. (Color online) Amplitudes (color mapping) and phase distribution of order parameters for an isotropic $s$-wave pairing state for a $d_{x z}$ orbital [(a) and (b)], a $d_{y z}$ orbital [(c) and (d)], and a $d_{x y}$ orbital [(e) and (f)], respectively. The phase distribution of order parameters has been mapped to a vector field. The length of arrows represents the amplitude of order parameters.

following:

$$
\begin{aligned}
H_{0}= & \sum_{k} \psi^{\dagger}(k) M(k) \psi(k), \\
M(k)= & K_{0}+K_{1} e^{i k_{x}}+C_{4 z} K_{1} C_{4 z}^{3} e^{i k_{y}} \\
& +C_{2 z} K_{1} C_{2 z} e^{-i k_{x}}+C_{4 z}^{3} K_{1} C_{4 z} e^{-i k_{y}} \\
& +K_{2} e^{i\left(k_{x}+k_{y}\right)}+C_{4 z} K_{2} C_{4 z}^{3} e^{i\left(-k_{x}+k_{y}\right)} \\
& +C_{2 z} K_{2} C_{2 z} e^{i\left(-k_{x}-k_{y}\right)}+C_{4 z}^{3} K_{2} C_{4 z} e^{i\left(k_{x}-k_{y}\right)},
\end{aligned}
$$

where $\psi^{\dagger}(k)=\left[a_{x z}^{\dagger}(k), a_{y z}^{\dagger}(k), a_{x y}^{\dagger}(k)\right]$ and the fourfold rotation is carried out by one of the generators of $D_{4}$ group:

$$
C_{4 z}=\left(\begin{array}{rrr}
0 & -1 & 0 \\
1 & 0 & 0 \\
0 & 0 & 1
\end{array}\right)
$$

The irreducible hopping subsets [40] (in eV) corresponding to on-site atomic energies, hopping along $\hat{x}$, and $\hat{x}+\hat{y}$ directions 
are

$$
\begin{aligned}
K_{0} & =\operatorname{diag}(-\mu,-\mu, 0.4-\mu), \\
K_{1} & =\left(\begin{array}{llr}
0.05 & 0.00 & -0.20 \\
0.00 & 0.01 & 0.00 \\
0.20 & 0.00 & 0.20
\end{array}\right), \\
K_{2} & =\left(\begin{array}{rrr}
0.02 & 0.01 & 0.10 \\
0.01 & 0.02 & 0.10 \\
-0.10 & -0.10 & 0.20
\end{array}\right) .
\end{aligned}
$$

For simplicity, the spin indices have been dropped. Instead of going along the boundary of the irreducible BZ, an alternative path has been used to show the band structure with dominating orbital weights in Fig. 2(a). The projected density of states (PDOS) reveals strongly-hybridized bands, which are composed of $d_{x z}$ and $d_{y z}$ orbitals along the off-diagonal line of the extend BZ below the Fermi level. The Fermi surface [Fig. 2(b)], obtained with a chemical potential $\mu=0.312 \mathrm{eV}$ corresponding to a filling factor $n=4.23$, has four electron pockets that do not have any $\mathrm{SC}$ gap node in case of anisotropic $s$ - and $d_{x^{2}-y^{2}}$-wave pairing sates. The absence of electron or hole pockets at $\Gamma$ point is consistent with the experimental observation [12].

The Hamiltonian in Eq. (1) can be diagonalized by conducting the Bogoliubov-Valatin transformation [41,42] containing $t_{2 g}$ orbital degrees of freedom as

$$
a_{i \alpha \sigma}=\sum_{\epsilon_{n \uparrow}>0} u_{i \alpha \sigma \sigma}^{n} \gamma_{n \sigma}+\bar{\sigma} v_{i \alpha \sigma \bar{\sigma}}^{n *} \gamma_{n \bar{\sigma}}^{\dagger},
$$

where the quasiparticle creation operator $\gamma_{n \sigma}^{\dagger}$ is the ladder operator of the eigenspectrum of the Hamiltonian, which satisfies $\left[H, \gamma_{n \sigma}^{\dagger}\right]_{-}=\epsilon_{n \sigma} \gamma_{n \sigma}^{\dagger}$. The diagonal condition of the Hamiltonian is the $\mathrm{BdG}$ equation

$$
\begin{gathered}
\sum_{j, \beta}\left[\begin{array}{cc}
\tilde{h}_{\uparrow \uparrow}(i \alpha, j \beta) & \tilde{\Delta}_{\uparrow \downarrow}(i \alpha, j \beta) \\
\tilde{\Delta}_{\uparrow \downarrow}^{*}(i \alpha, j \beta) & -\tilde{h}_{\downarrow \downarrow}^{*}(i \alpha, j \beta)
\end{array}\right]\left[\begin{array}{c}
u_{j \beta \uparrow \uparrow}^{n} \\
v_{j \beta \downarrow \uparrow}^{n}
\end{array}\right] \\
=\epsilon_{n \uparrow}\left[\begin{array}{c}
u_{i \alpha \uparrow \uparrow}^{n} \\
v_{i \alpha \downarrow \uparrow}^{n}
\end{array}\right],
\end{gathered}
$$

where $\tilde{h}_{\sigma \sigma}(i \alpha, j \beta)=\tilde{t}_{\sigma \sigma}(i \alpha, j \beta)-\mu \delta_{i j} \delta_{\alpha \beta}$ and the order parameters defined on different orbitals are

$$
\begin{aligned}
& \tilde{\Delta}_{\uparrow \downarrow}(i \alpha, j \beta) \\
& =-\frac{V_{\uparrow \downarrow}(i \alpha, j \beta)}{2} \sum_{\epsilon_{n \uparrow}>0,<0} u_{i \alpha \uparrow \uparrow}^{n} v_{j \beta \downarrow \uparrow}^{n *} \tanh \left(\frac{\epsilon_{n \uparrow}}{2 k_{B} T}\right) .
\end{aligned}
$$

Equations (3) and (15) give a nontrivial winding boundary condition to quasiparticle amplitudes as

$$
\left[\begin{array}{c}
u_{i+\lambda, \alpha \uparrow \uparrow}^{n} \\
v_{i+\lambda, \alpha \downarrow \uparrow}^{n}
\end{array}\right]=\left[\begin{array}{c}
e^{i \frac{\pi}{2} \mathcal{N}_{v}\left[\lambda_{x} \lambda_{y}+\frac{1}{N}\left(\lambda_{x} i_{y}-\lambda_{y} i_{x}\right)\right]} u_{i \alpha \uparrow \uparrow}^{n} \\
e^{-i \frac{\pi}{2} \mathcal{N}_{v}\left[\lambda_{x} \lambda_{y}+\frac{1}{N}\left(\lambda_{x} i_{y}-\lambda_{y} i_{x}\right)\right]} v_{i \alpha \downarrow \uparrow}^{n}
\end{array}\right] .
$$

The order parameters are calculated by BdG equation self-consistently with the above boundary condition, which is assigned to the matrix element $\tilde{h}_{\sigma \sigma}(i \alpha, j \beta)$ and $\tilde{\Delta}_{\uparrow \downarrow}(i \alpha, j \beta)$ for $\mathcal{N}_{v}=1$. The self-consistent calculation starts with arbitrarily distributed order parameters and the iteration is performed with a convergence criterion that the order parameters have a relative difference less that $10^{-3}$ between two consecutive steps. The particle density is calculated via quasiparticle wave functions as

$$
\begin{aligned}
& \left\langle n_{i \alpha \uparrow}\right\rangle=\frac{1}{2} \sum_{\epsilon_{n \uparrow}>,<0}\left|u_{i \alpha \uparrow \uparrow}^{n}\right|^{2}\left[1-\tanh \left(\frac{\epsilon_{n \uparrow}}{2 k_{B} T}\right)\right], \\
& \left\langle n_{i \alpha \downarrow}\right\rangle=\frac{1}{2} \sum_{\epsilon_{n \uparrow} \uparrow,<0}\left|v_{i \alpha \downarrow \uparrow}^{n}\right|^{2}\left[1+\tanh \left(\frac{\epsilon_{n \uparrow}}{2 k_{B} T}\right)\right] .
\end{aligned}
$$

The energy spectrum of the quasiparticle, i.e., the LDOS at site $i$ for orbital $\alpha$ is calculated via

$$
\begin{aligned}
\rho_{i \alpha}(\epsilon)= & \frac{1}{M_{x} M_{y}} \sum_{\vec{k} \in F B Z} \sum_{\epsilon_{n \uparrow}>,<0}\left|u_{i \alpha \uparrow \uparrow}^{n}\right|^{2} \delta\left[\epsilon-\epsilon_{n \uparrow}(\vec{k})\right] \\
& +\left|v_{i \alpha \downarrow \uparrow}^{n}\right|^{2} \delta\left[\epsilon+\epsilon_{n \uparrow}(\vec{k})\right]
\end{aligned}
$$

where the supercell method has been used [43] for $M_{x}=$ $M_{y}=10$. The Lorentzian smearing method is used to visualize the LDOS with a broadening width $\sigma=0.001$. All the selfconsistent calculations are performed on a $28 \times 28$ lattice at temperature $T=0.1 \mathrm{~K}$.

Calculation of magnetic exchange couplings shows that the leading pairing instability comes from the intraorbital pairing contribution, whereas the interorbital components are found to be significantly small [39]. Consequently, only an intraorbital pairing potential is considered in our numerical calculations. The SC gap function for a multiorbital superconductor is generally defined in momentum space as

$$
\Delta_{\alpha \beta}^{i}(\vec{k})=g^{i}(\vec{k}) \Gamma_{\alpha \beta}\left(i \sigma_{2}\right),
$$

where $g^{i}(\vec{k})$ is the basis of the irreducible unitary representations of $D_{4}$ point group, $i \sigma_{2}$ defines a tensor state for singlet pairing, and $\Gamma_{\alpha \beta}$ is the orbital basis for $D_{4}$ transformation. The transformation properties of the band structure determine all the symmetry transformations of SC order parameters [39,45]. Another reason that the interorbital pairing has been omitted in our calculation is that only if $\Gamma_{\alpha \beta}$ transform according to

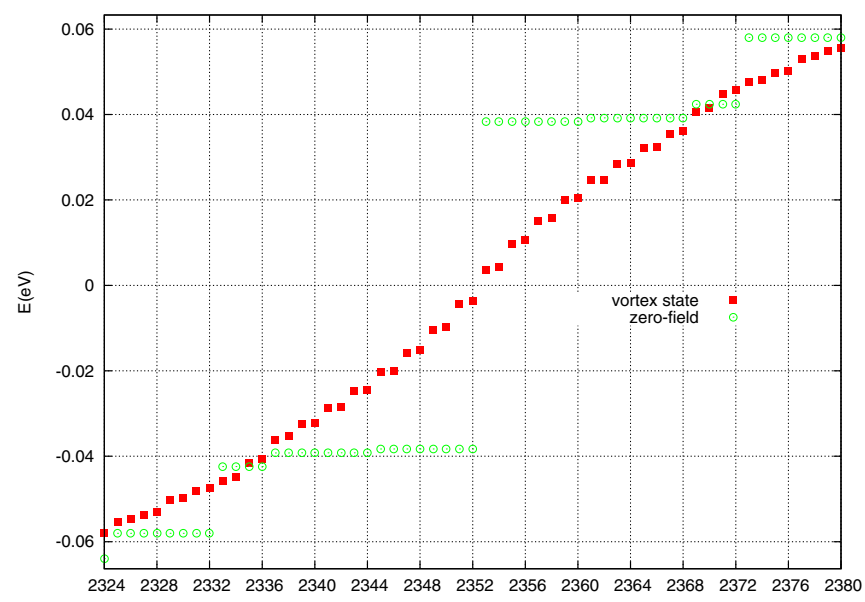

FIG. 5. (Color online) Eigenvalues of BdG equation at around the Fermi level in the cases of isotropic $s$-wave pairing state for zero-field states, shown in green circles, and vortex states, shown in red squares, respectively. The eigenvalues are plotted in an ascending sequence in the horizontal axis. 


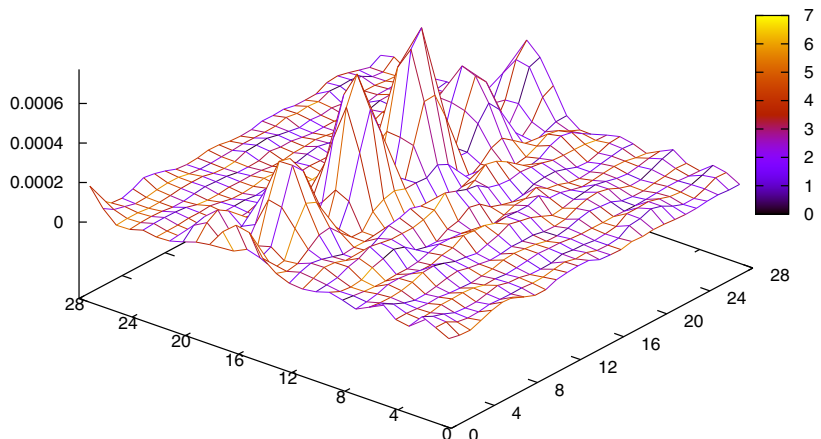

(a)

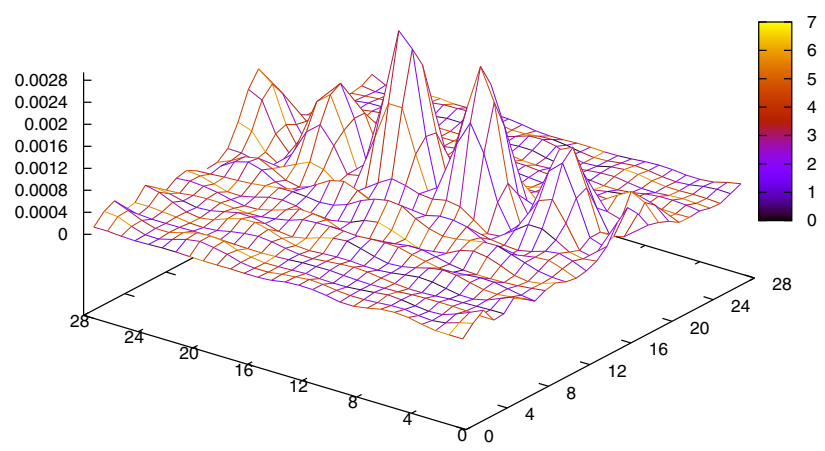

(c)

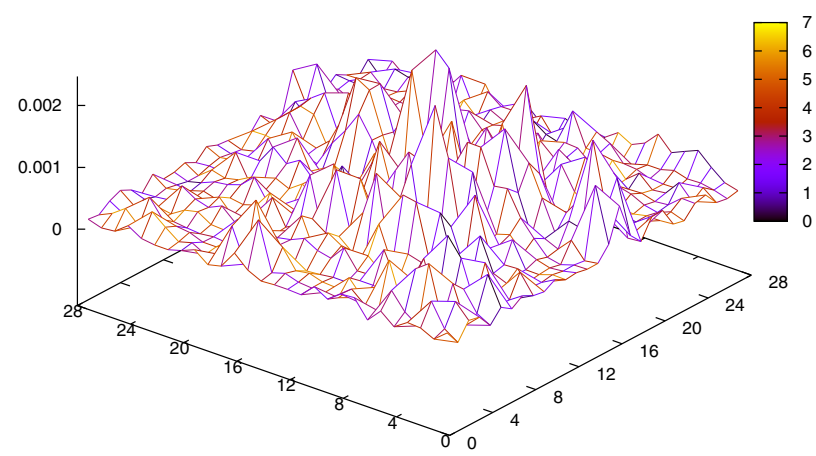

(e)

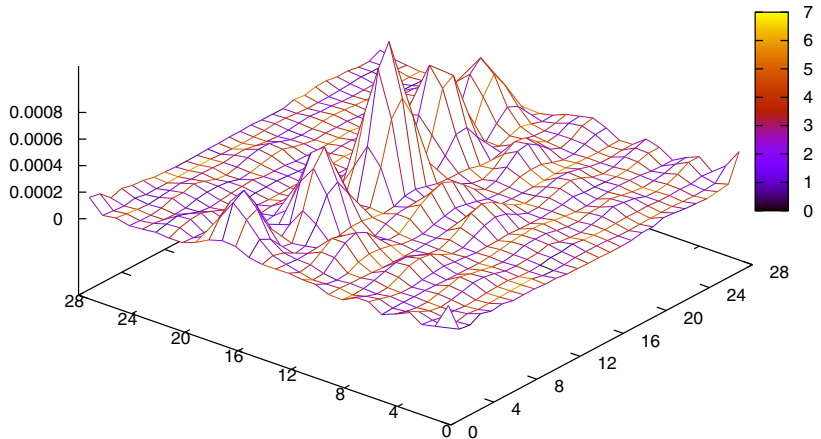

(b)

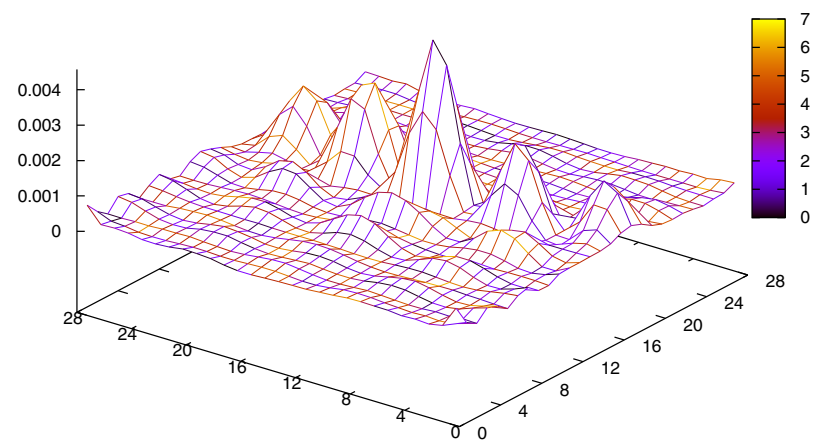

(d)

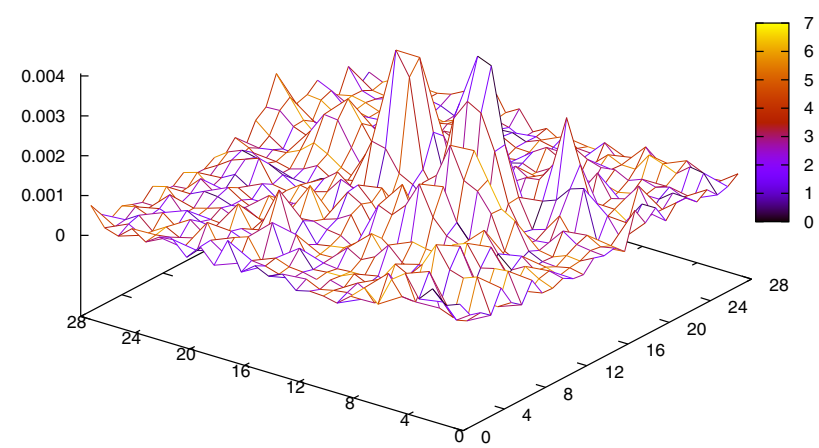

(f)

FIG. 6. (Color online) Amplitudes and phases (color mapping) of quasiparticle wave functions $u_{i \alpha \uparrow \uparrow}^{n}$ and $v_{i \alpha \downarrow \uparrow}^{n}$ for isotropic $s$-wave pairing symmetry of index $n=2353$ for a $d_{x z}$ orbital [(a) and (b)], a $d_{y z}$ orbital [(c) and (d)], and a $d_{x y}$ orbital [(e) and (f)], respectively.

$A_{1 g}$ representation, then the symmetry of the pairing state can be exclusively determined by its spatial component $g^{i}(\vec{k})$, such that the calculated vortex sate has a classification of Table I. For isotropic $s$-wave pairing,

$$
V_{\uparrow \downarrow}(i \alpha, j \alpha)=-g_{0} \delta_{i j},
$$

for anisotropic $s$-wave pairing,

$$
\begin{aligned}
V_{\uparrow \downarrow}(i \alpha, j \alpha)= & -\frac{g_{1}}{4}\left(\delta_{i+\hat{x}+\hat{y}, j}+\delta_{i-\hat{x}+\hat{y}, j}\right. \\
& \left.+\delta_{i-\hat{x}-\hat{y}, j}+\delta_{i+\hat{x}-\hat{y}, j}\right),
\end{aligned}
$$

and for $d_{x^{2}-y^{2}}$-wave pairing,

$$
V_{\uparrow \downarrow}(i \alpha, j \alpha)=-\frac{g_{2}}{2}\left(\delta_{i+\hat{x}, j}+\delta_{i+\hat{y}, j}+\delta_{i-\hat{x}, j}+\delta_{i-\hat{y}, j}\right),
$$

where $g_{0,1,2}$ are pairing amplitudes for each pairing symmetry. Figure 3 shows the Fermi velocity $\hbar \vec{v}_{n}(\vec{k})=\nabla_{\vec{k}} \epsilon_{n}(\vec{k})$, which is used to determine the pairing potential. In order to mimic the intermediate coupling cases for FeSe [10] and $A_{y} \mathrm{Fe}_{2-x} \mathrm{Se}_{2}$ ( $A=\mathrm{K}, \mathrm{Rb}$, or $\mathrm{Cs}$ ) [44] superconductors whose coherent length $\xi=\frac{\hbar v_{F}}{\pi \Delta(0)}$ ranges from $4 a$ to $12 a$, where $a$ is the lattice constant, the maximum pairing amplitudes are taken to be $g_{0}=0.62, g_{1}=2.60$, and $g_{2}=1.28$, respectively, which results in two $\mathrm{SC}$ order parameters $(\mathrm{eV})$ due to the orbital anisotropy in the zero-field case as for an isotropic $s$ wave:

$$
\left|\Delta_{x z, y z}^{s}(0)\right|=0.047, \quad\left|\Delta_{x y}^{s}(0)\right|=0.026 ;
$$

for an anisotropic $s$ wave:

$$
\left|\Delta_{x z, y z}^{\text {anis. } s}(0)\right|=0.048, \quad\left|\Delta_{x y}^{\text {anis. } s}(0)\right|=0.023 ;
$$



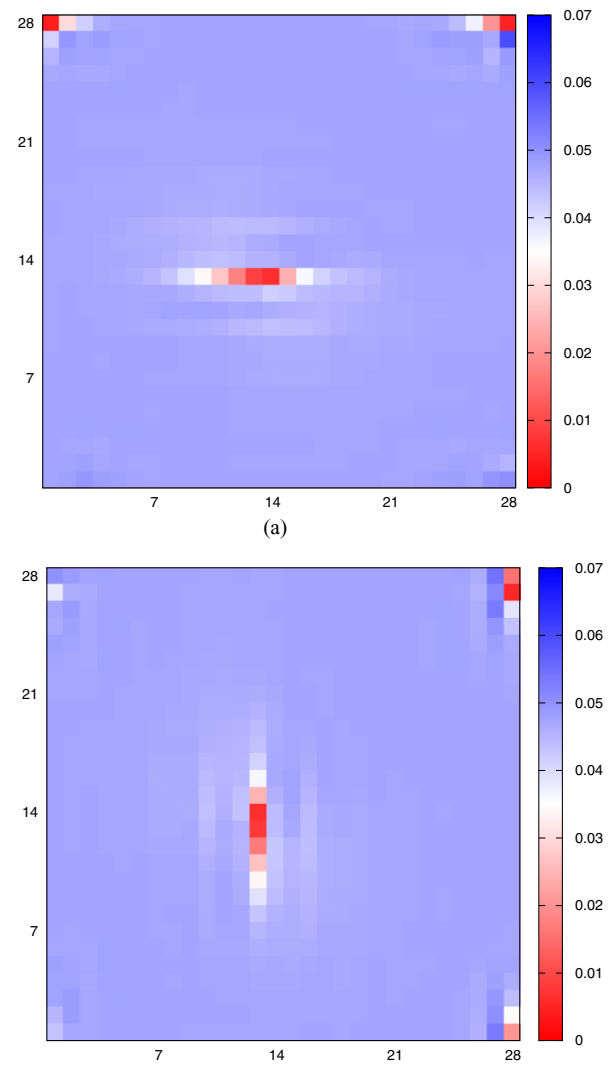

(c)

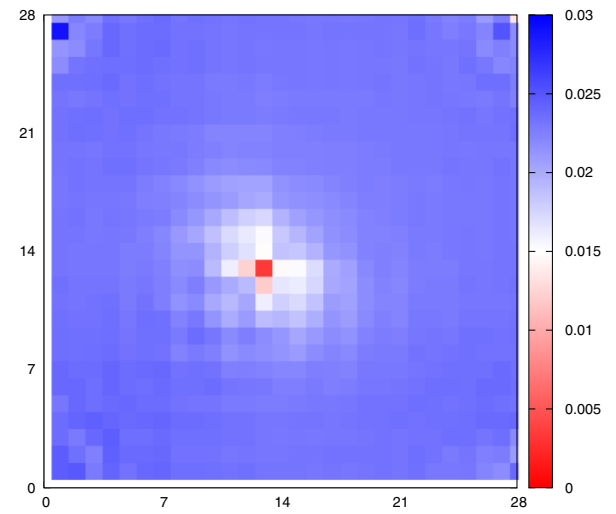

(e)

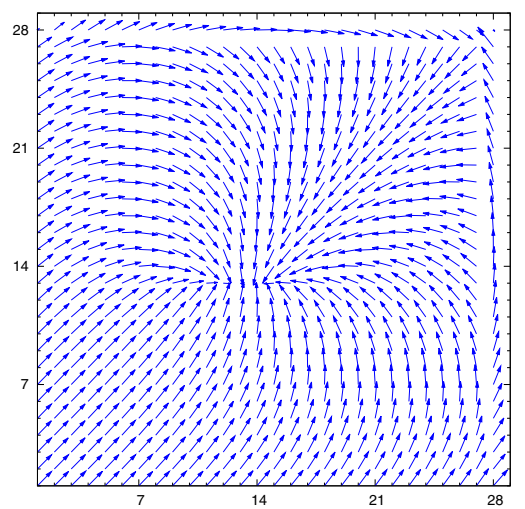

(b)

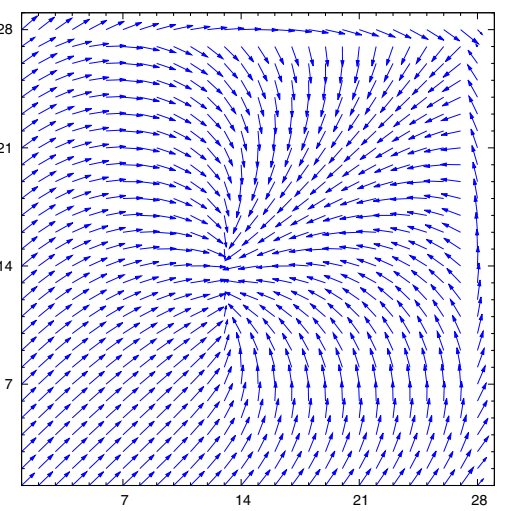

(d)

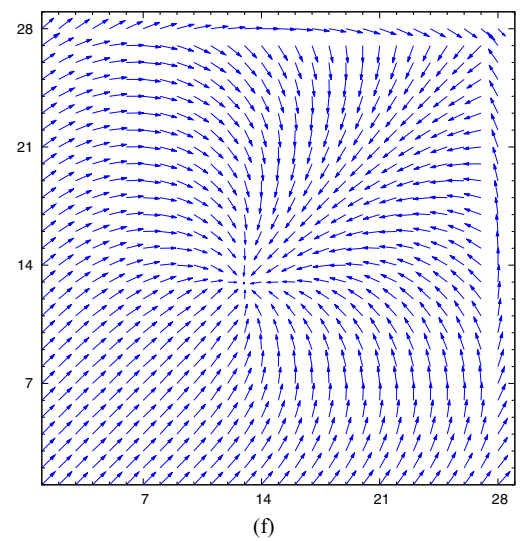

FIG. 7. (Color online) Amplitudes (color mapping) and phase distribution of anisotropic $s$-wave pairing bonds along the $\hat{x}+\hat{y}$ direction for a $d_{x z}$ orbital [(a) and (b)], a $d_{y z}$ orbital [(c) and (d)], and a $d_{x y}$ orbital [(e) and (f)], respectively. Results of pairing bonds along $-\hat{x}+\hat{y}$, $-\hat{x}-\hat{y}$, and $\hat{x}-\hat{y}$ directions are the same with these results.

and for a $d_{x^{2}-y^{2}}$ wave:

$$
\left|\Delta_{x z, y z}^{d_{x^{2}-y^{2}}}(0)\right|=0.048, \quad\left|\Delta_{x y}^{d_{x^{2}-y^{2}}}(0)\right|=0.025 .
$$

\section{RESULTS AND DISCUSSION}

The vortex structures for an isotropic $s$-wave pairing state are shown in Fig. 4 for different orbitals, respectively. The vortex states exhibit orbital anisotropy. For $d_{x z}$ and $d_{y z}$ orbitals, the amplitudes have two plateaus with a difference of about $0.005 \mathrm{eV}$ along the $\hat{y}$ and $\hat{x}$ directions on both sides of the core region and the pinning center deviates slightly from the center of the magnetic unit cell. The phase distribution shows a winding number $\mathcal{W}=1$, such that the symmetry subgroup of the vortex structure is $G_{5}$ [32]. The winding structure of the $s$-wave vortex, as mapped to a vector field, has a sink-type core center. Figure 5 shows the eigenvalues obtained from vortex and zero-field states, where it has been found there are 16 in-gap eigenstates for both positive and negative eigenvalues. We examine the behavior of the quasiparticle wave function $u_{i \alpha \uparrow \uparrow}^{n}$ and $v_{i \alpha \downarrow \uparrow}^{n}$ and it turns out that all the 32 in-gap states are extended to the entire magnetic unit cell (Fig. 6, eigenstate $\left.\left|\epsilon_{2353 \uparrow}\right\rangle\right)$. The orbital anisotropy appears again as for the $d_{x z}$ and $d_{y z}$ orbitals, the wave function extends to the $\hat{x}$ and $\hat{y}$ direction because the spatial orientation of the $d$-orbital harmonics, whereas for a $d_{x y}$ orbital, the spreading of the 


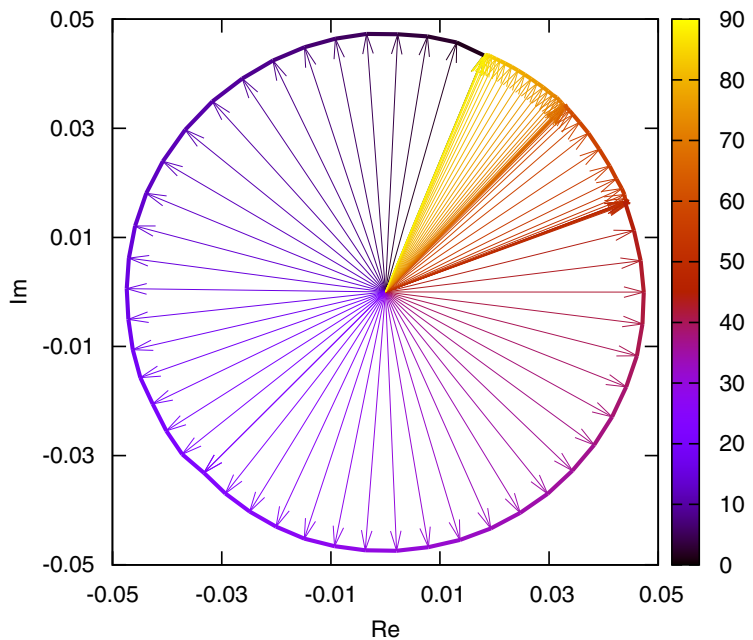

(a)

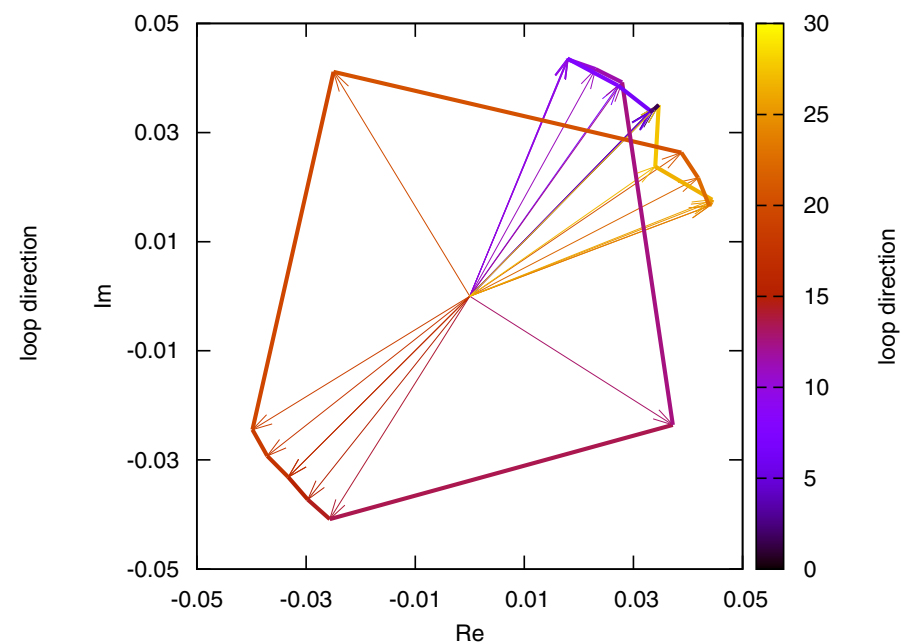

(b)

FIG. 8. (Color online) Phase mapping onto a complex plane of an anisotropic $s$-wave pairing bond along $\hat{x}+\hat{y}$ direction. The loop around the center of the magnetic unit cell (a) is $(25,3) \rightarrow(25,25) \rightarrow(3,25) \rightarrow(3,3) \rightarrow(25,3)$ and around a corner of magnetic unit cell $(\mathrm{b})$ is $(3,1) \rightarrow(3,3) \rightarrow(1,3) \rightarrow(28,3) \rightarrow(25,3) \rightarrow(25,1) \rightarrow(25,28) \rightarrow(25,25) \rightarrow(28,25) \rightarrow(1,25) \rightarrow(3,25) \rightarrow(3,28) \rightarrow(3,1)$. The loop direction has been shown by color mapping of each step.

wave function is symmetric in $\hat{x}$ and $\hat{y}$ directions. These extended wave functions amount to a large scale variation of order parameters within the entire magnetic unit cell and consequently a relatively large vortex core region.

The structures of anisotropic $s$-wave vortices are shown in Fig. 7. The core regions of $d_{x z / y z}$ orbital vortices are not a geometric point any more. Instead, they have been stretched along $\hat{x}$ and $\hat{y}$ directions due to the fact that although the pairing bonds are defined on next-nearest-neighbor sites, the electrons forming Cooper pairs come from distinguishable oriented orbitals. The symmetry subgroup of anisotropic $s$ wave vortices is still $G_{5}$, but the orbital asymmetry results in a line-type topological defect for $d_{x z / y z}$ orbital vortices, whereas the $d_{x y}$ orbital vortex is still of a sink type. One special fact worth noting is that there is a suppression of order parameters at corners of magnetic unit cell, which also exists for pairing bond

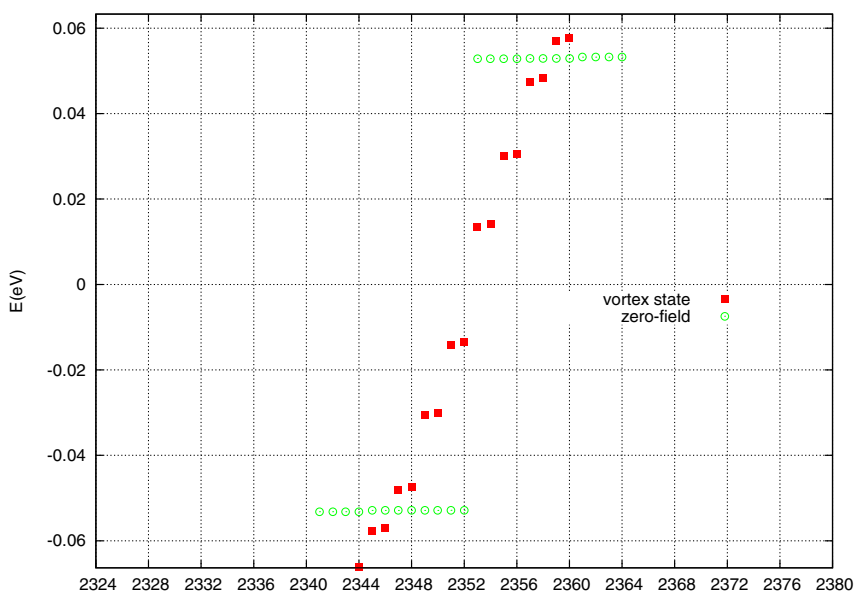

FIG. 9. (Color online) Eigenvalues of BdG equation at around the Fermi level in the cases of an anisotropic $s$-wave pairing state for zero-field states, shown in green circles, and vortex states, shown in red squares, respectively. along $-\hat{x} \pm \hat{y}$ and $\hat{x}-\hat{y}$ directions. In order to understand the physical origin of this phenomena, we examine the phase variation along two loops around the center and corner of the magnetic unit cell, respectively. The loop around the corner is well-defined in the order parameter space because the nontrivial winding periodic boundary condition (18) has been applied. Since the homotopy group of order parameter space of a vortex state is $\pi_{1}[U(1)]=\mathbb{Z}$ and that the winding number $\mathcal{N}_{v}=1$ has been fixed when the self-consistent calculation is carried out, we expect that the variation along the loop around the corner is definitely not homotopic, equivalent to that around the vortex at the center. Figure 8(a) shows the phase variation around the vortex core, where the phases change slowly on a number of lattice sites at the very beginning of the loop as shown in Fig. 7(a) in the vicinity of site $(25,3)$. We have deliberately chosen a loop far away from the core region, since a stable topological defect always leaves its signature anywhere arbitrarily away from it [1]. However, the phase variation of order parameters around the corner of magnetic unit cell exhibits some turning-back points, from which the clockwise increments contribute a negative phase winding. Therefore the total winding around the corner is zero, which proves that the suppression of order parameters at the corners of the magnetic unit cell is not a vortex. A detailed analysis about the phase difference on each lattice site shows that such singularities at the corners are actually caused by the discontinuity of boundary conditions of the wave function of each orbital when the calculation is carried out on an $N_{x} \times N_{y}$ lattice. From Eq. (18), we know that the variation of boundary conditions along the $\hat{x}$ direction for adjacent $\left(\lambda_{x}=1, \lambda_{y}=0\right)$ magnetic unit cells is $e^{i K N_{x} i_{y}}$, and it will come back to $e^{i\left(K N_{x}+2 \pi\right)}$ when the condition $i_{y}=4 N_{y}+1$ is satisfied. It is obvious that such a condition cannot be realized in a numerical calculation for any given $N_{y}$, therefore the discontinuity, which can be regarded as an impurity induced by a winding boundary condition, cannot be avoided. The impurity nature of these singularities can also be recognized as 


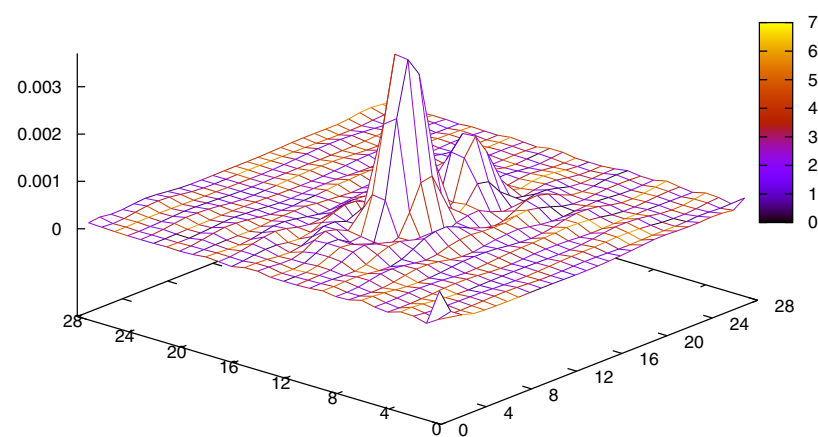

(a)

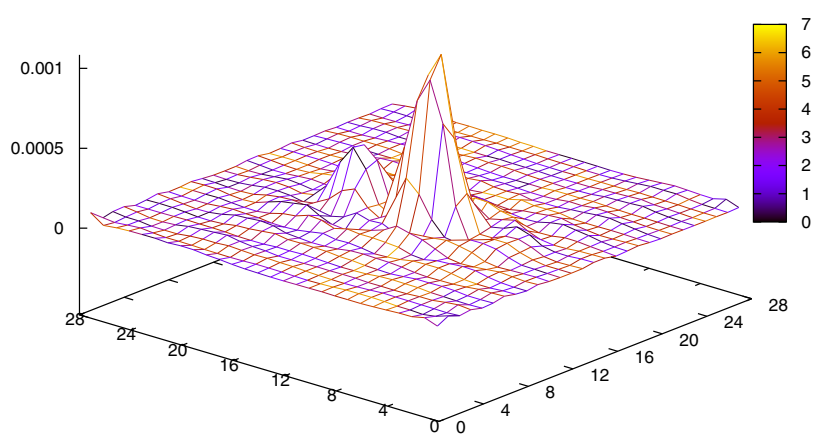

(c)

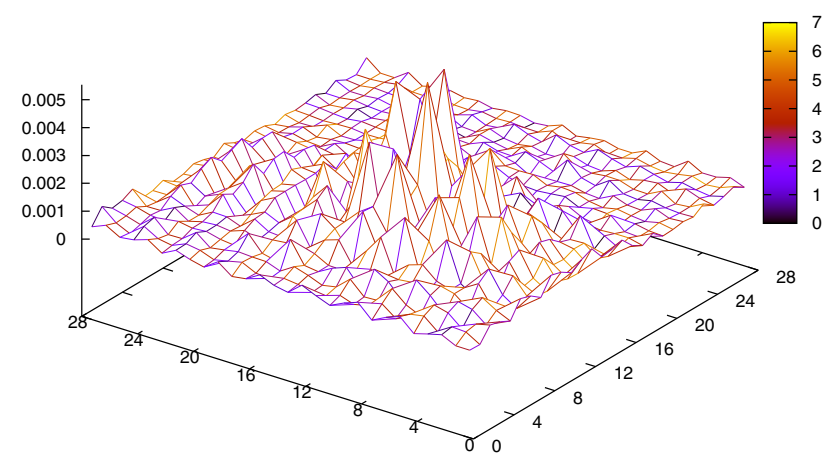

(e)

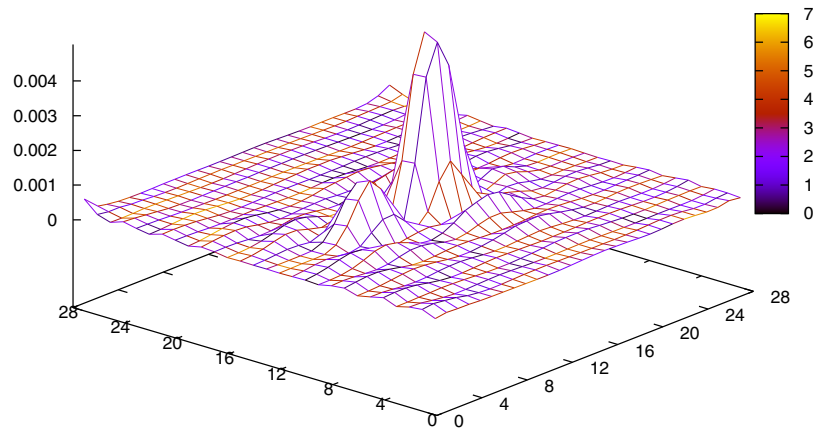

(b)

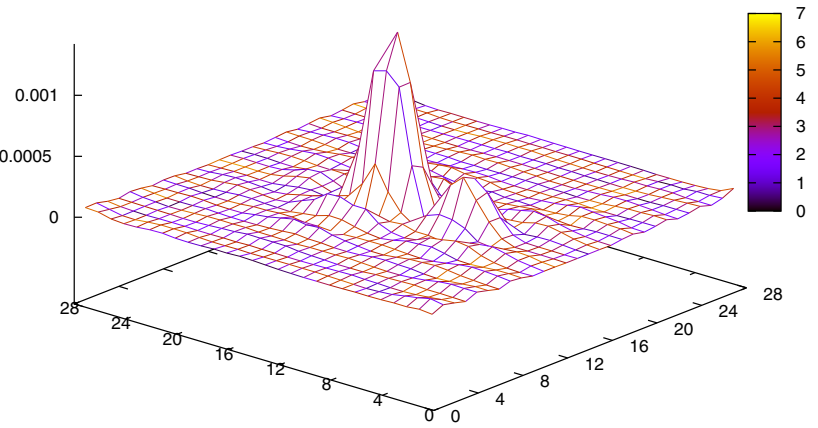

(d)

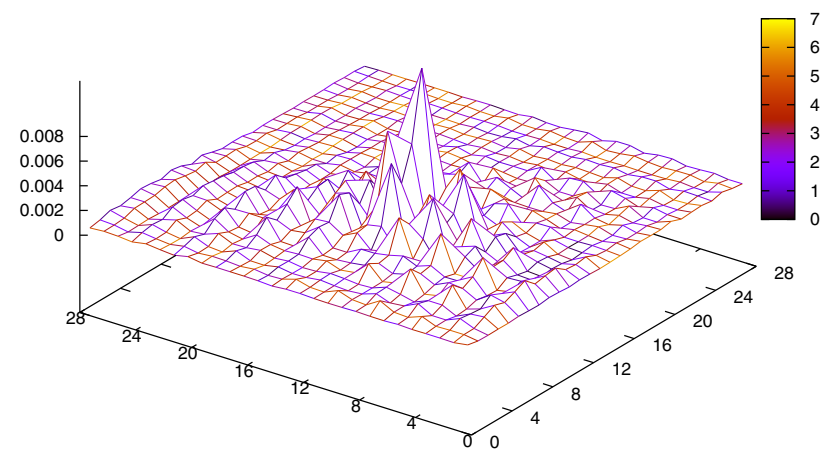

(f)

FIG. 10. (Color online) Amplitudes and phases (color mapping) of quasiparticle wave functions $u_{i \alpha \uparrow \uparrow}^{n}$ and $v_{i \alpha \downarrow \uparrow}^{n}$ for anisotropic $s$-wave pairing state of index $n=2353$ for $d_{x z}$ orbital (a) and (b), $d_{y z}$ orbital (c) and (d), and $d_{x y}$ orbital (e) and (f), respectively.

a suppression of the order parameters occurring on a single site at the corners, which is different from a genuine vortex having an effective core region. We also noted that such a singularity does not exist for $\mathcal{N}_{v}=4$, but in this case the vortex states cannot be classified by invariant subgroups of the magnetic translation group, which is originally aimed at describing the Abrikosov lattice for $\mathcal{N}_{v}=1$. There are 12 in-gap eigenstates, as shown in Fig. 9, which are located symmetrically on both sides of the Fermi level. The wave functions of these states are typically localized for $d_{x z / y z}$ orbitals and extended for $d_{x y}$ orbital, as shown in Fig. 10 for an eigenstate $\left|\epsilon_{2353}\right\rangle$. It has been observed that the wave functions for each orbital show particle-hole asymmetry. Although the difference of vortices between isotropic $s$ and anisotropic $s$-wave pairing states has been observed from the hitherto results, such a difference may rely on the limitation of our model calculation in that since the Hamiltonian is defined on a site-orbital representation, there is no well-defined $k$-space energy cutoff in the vicinity of the Fermi level for the attractive pairing potential. Therefore pairing electrons may come from a region far away from the four electron pockets. Consequently, the absence of a pocket at $\Gamma$ point may induce ambiguity for an anisotropic $s$-wave pairing state in a framework of BCS-type pairing scheme.

The results of $d_{x^{2}-y^{2}}$-wave vortices are different from the $A_{1 g}$ vortices discussed above in many aspects. The orbital anisotropy dominates the vortex structures. Figures 11 and 12 show the amplitudes and phase distribution of $d_{x^{2}-y^{2}}$-wave pairing bonds for each orbital. It has been pointed out in the previous section that the symmetry of the band structure gives constraints to the symmetry of the pairing states. A strong hybridization of $d_{x z}$ and $d_{y z}$ orbitals, as shown in PDOS in Fig. 2, results in a redefined $d_{x^{2}-y^{2}}$-wave pairing state, as shown in Fig. 13, since the wave functions of these two orbitals transform under the action of generator $C_{4 z}$ as

$$
C_{4 z}\left|d_{x z}\right\rangle=\left|d_{y z}\right\rangle, \quad C_{4 z}\left|d_{y z}\right\rangle=-\left|d_{x z}\right\rangle
$$




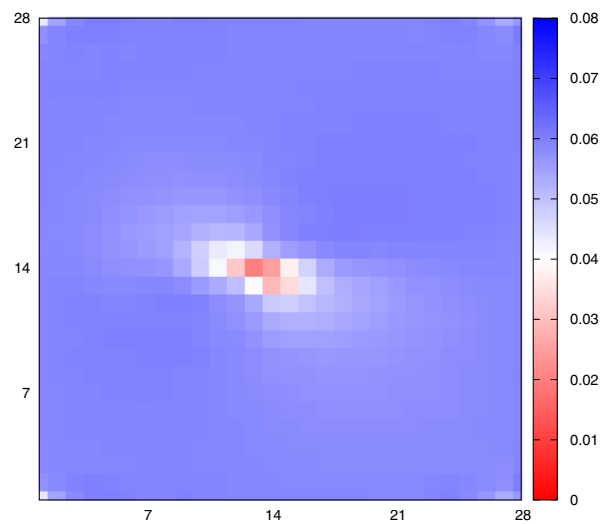

(a)

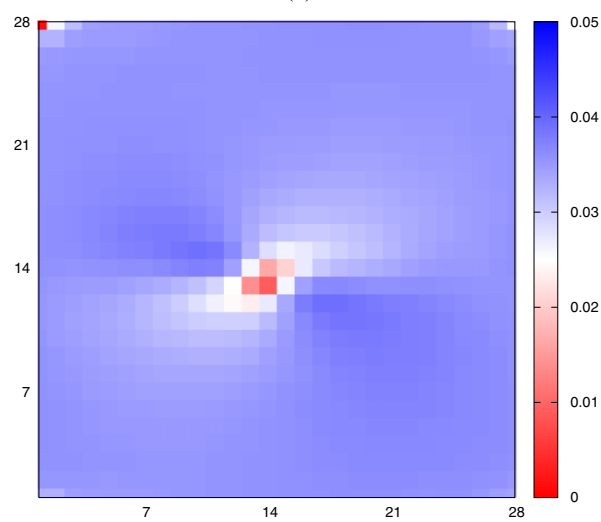

(c)

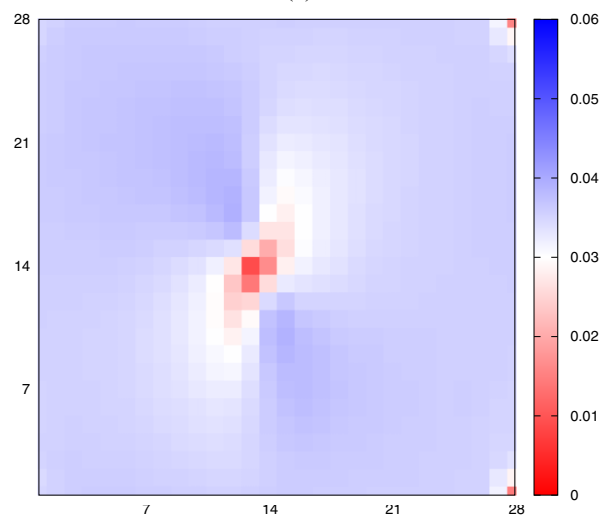

(e)

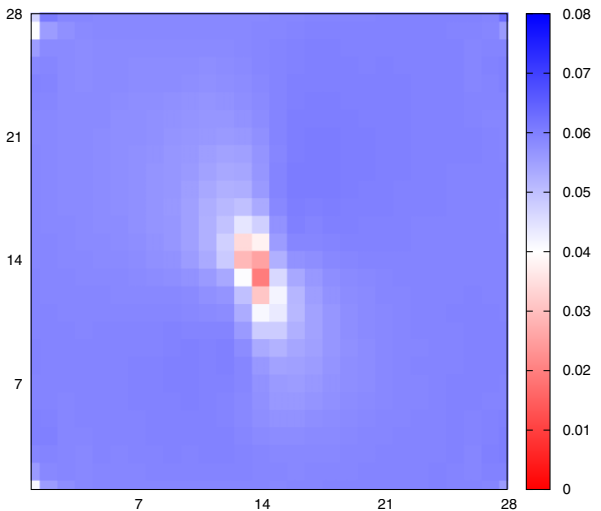

(g)
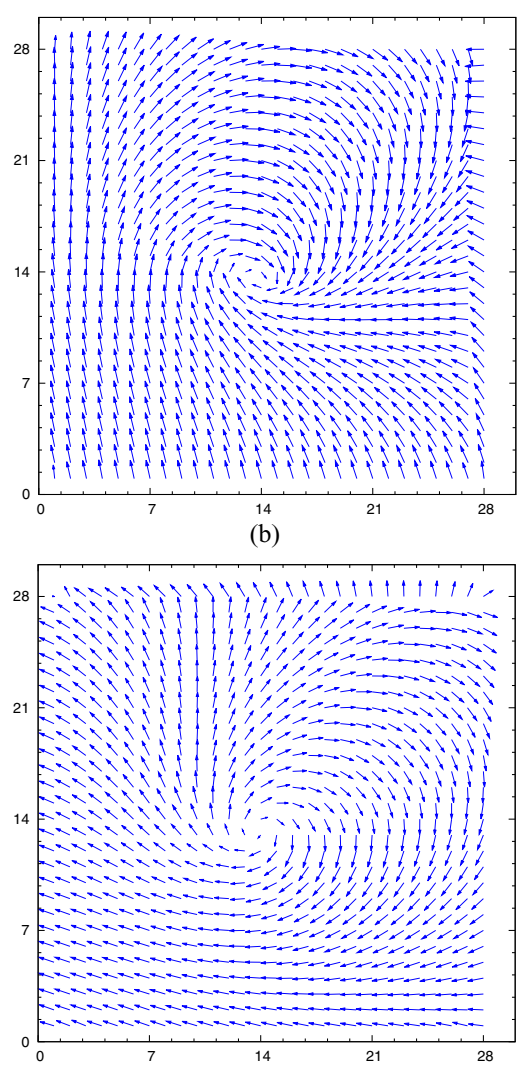

(d)

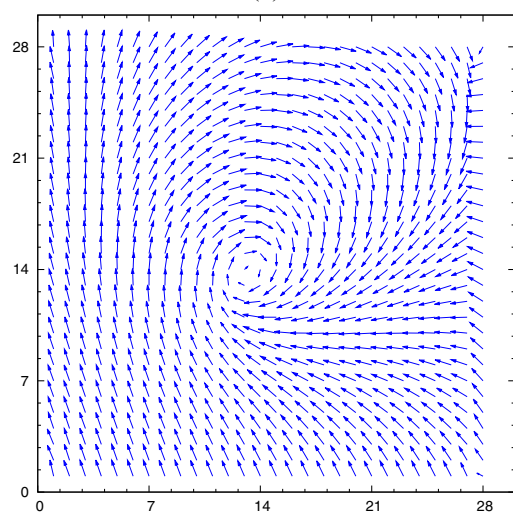

(f)

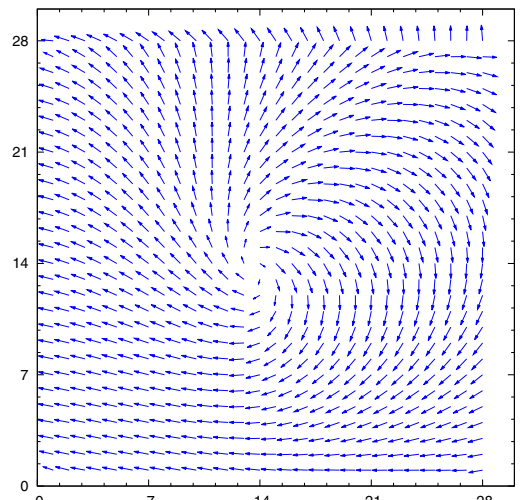

(h)

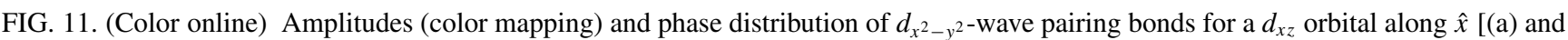
(b)] and $\hat{y}\left[(\mathrm{c})\right.$ and (d)] and for a $d_{y z}$ orbital along $\hat{x}[(\mathrm{e})$ and (f)] and $\hat{y}[(\mathrm{~g})$ and (h)] directions, respectively. The results of pairing bonds along the other two directions of the next-nearest-neighbor site pairing are same with these results. 

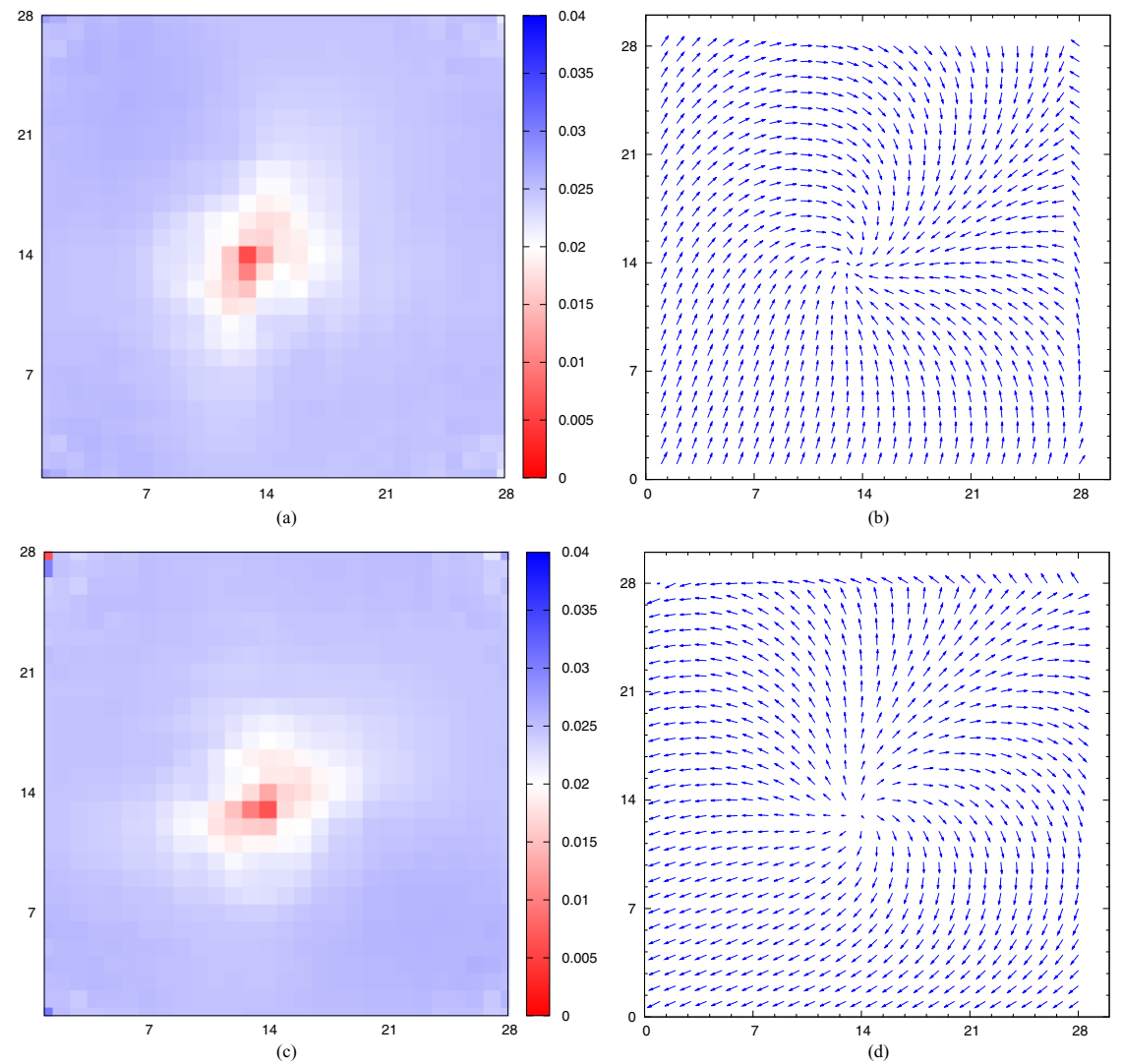

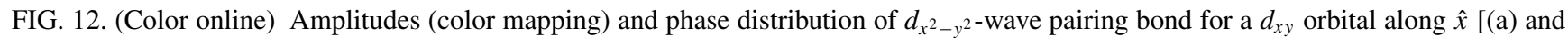
(b)] and $\hat{y}[(\mathrm{c})$ and (d)] directions, respectively. Results of pairing bonds along the other two directions of next-nearest site pairing are same with these results.

while the $d_{x y}$ orbital does not mix with them under such a transformation. Here, we give an example of the numerical results of the order parameters for each orbital on site $(3,3)$, as shown in Table II. In the zero-field case, a phase difference of $e^{i \pi}$ is observed between $\pi_{x}$ and $\pi_{y}$, and $\sigma_{x}$ and $\sigma_{y}$ bonds, which are defined on different orbitals, whereas in the vortex states, such a phase will undergo a gauge modification induced by the magnetic field. The winding structures shown in Figs. 11 and 12 for different orbitals share this common feature for all order parameters defined on the entire magnetic unit cell. For a $d_{x y}$ orbital, $G_{5}^{*}$ vortices, which are defined on pairing bonds $\Delta_{x y}(\hat{x})$ and $\Delta_{x y}(\hat{y})$ are of sink and source types, respectively, because the order parameters change sign as they transform according to $B_{1 g}$ irreducible unitary representation. In the presence of a magnetic field, the sign change of the $d_{x^{2}-y^{2}-}$ wave pairing symmetry along with the orbital-hybridized order parameters give rise to a $G_{6}^{*}$ winding structure for $d_{x z / y z}$ orbitals, which seems like a solenoidal vector field. Such phase difference has been observed between $\Delta_{x z}\left(\sigma_{x}\right)$ as shown in Fig. 11(b) and $\Delta_{y z}\left(\sigma_{y}\right)$ as shown in Fig. 11(h), and also between $\Delta_{x z}\left(\pi_{y}\right)$ as shown in Fig. $11(\mathrm{~d})$ and $\Delta_{y z}\left(\pi_{x}\right)$ as shown in Fig. 11(f). Among the seventeen (positive) in-gap states associated with the orbital-resolved $d_{x^{2}-y^{2}}$-wave vortices as shown in Fig. 14, the wave functions of eigenvalue $\left|\epsilon_{2353 \uparrow}\right\rangle$ for $d_{x z}$ and $d_{y z}$ orbitals, and $\left|\epsilon_{2354 \uparrow}\right\rangle$ for $d_{x y}$ orbital are shown in Fig. 15. The particle-hole asymmetry is evidently for $d_{x z}$ and $d_{y z}$ orbitals in that the bound states have three peaks for the particle part and two peaks for the hole part. The most localized vortex bound state has been observed for a $d_{x y}$ orbital for the particle part. The discontinuity of the phase distribution on the boundary of the magnetic unit cell is also observed in $d_{x^{2}-y^{2}}$-wave vortices due to the next-nearest-neighbor site pairing.

In order to have an understanding of the distinction between vortex states of different pairing symmetries, we compare the orbital-resolved LDOS along the off-diagonal line approaching the vortex core and then away from it. Figure 16(a) shows the results for an isotropic $s$ wave, where the vortices of the $d_{x z}$ and $d_{y z}$ orbitals pinning at site $(15,15)$ are characterized by symmetrically located two peaks, while the vortex of the $d_{x y}$ orbital shows single peak. The two peaks start to shrink towards the Fermi level from site $(7,7)$ and then transit back to the SC coherence peak at site $(19,19)$, therefore the isotropic $s$-wave vortices have a relative large core region. Another characteristic of $s$-wave vortices is that the LDOS shows no Landau oscillation due to on-site pairing. However, since the wave functions of all the in-gap states for both positive and negative eigenstates are not localized, such vortex states may not be favored in an FeSe superconductor. Additionally, the particle-hole symmetry protects the electron density from accumulating or diminishing in the vortex core region as shown in Figs. 17(a) and 17(b). 


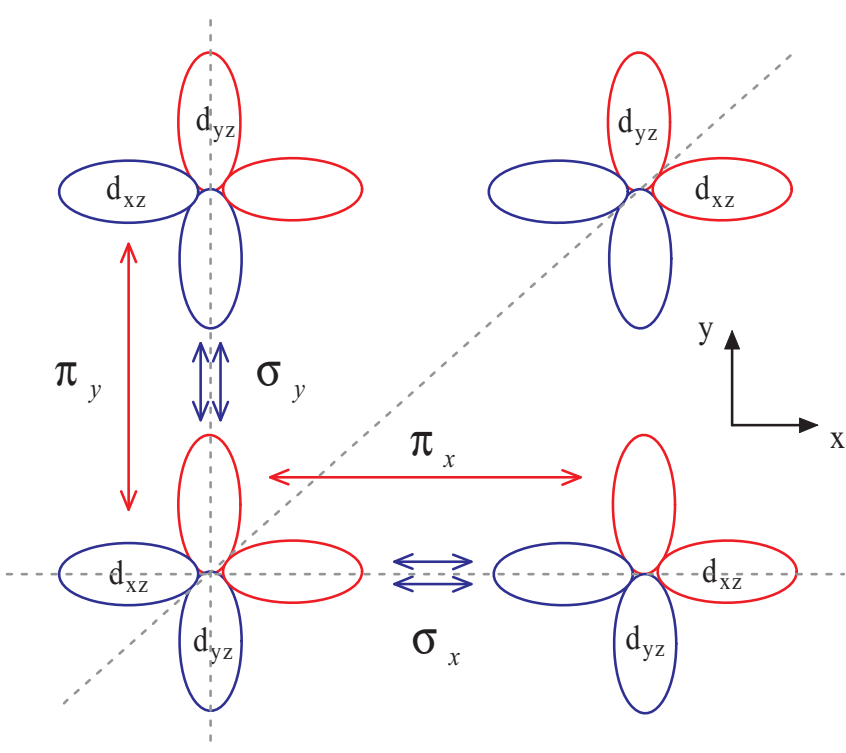

FIG. 13. (Color online) A schematic picture illustrates that the $d_{x^{2}-y^{2}}$-wave pairing state is re-defined between $d_{x z}$ and $d_{y z}$ orbitals due to the fourfold rotational symmetry. The red and blue color indicate positive and negative signs of orbital wave functions. The long and short double-headed arrows corresponding to $\pi$ and $\sigma$ pairing bonds along $\hat{x}$ and $\hat{y}$ directions show the exchange of orbital states under $C_{4 z}$ rotation.

For anisotropic $s$-wave vortices, an oscillation in LDOS for the $d_{x y}$ orbital has been observed, as shown in Fig. 16(b). The LDOS at the Fermi level varies alternately from zero at site $(3,3)$ to a finite value, and then oscillates until being stabilized at the core center. At site $(13,13)$ and $(14,14)$, the core states always manifest themselves as double peaks, which is different from the results of isotropic $s$ and $d_{x^{2}-y^{2}}$-wave vortices. Such an alternating appearance of the bound states at the Fermi level may come from the fact that for $d_{x z}$ and $d_{y z}$ orbitals, as shown in Fig. 17(c), there are charge density accumulations, while for $d_{x y}$ the orbital electron density is suppressed inside the core region, as shown in Fig. 17(d).

Finally, Fig. 16(c) shows the LDOS of $d_{x^{2}-y^{2}}$-wave vortices. It has been found that for $d_{x z / y z}$ orbitals, the vortex bound states are exactly localized at site $(14,14)$, with a stable SC coherence located at around $\pm 0.05 \mathrm{eV}$, and for the $d_{x y}$ orbital the core region includes site $(13,13)$. Similarly to the cases of anisotropic $s$-wave vortices, charge accumulation on the

TABLE II. Values (in $10^{-1} \mathrm{eV}$ ) of orbital-resolved $d_{x^{2}-y^{2}}$-wave pairing order parameters (pairing bonds) $\pi_{x, y}$ and $\sigma_{x, y}$ as defined in Fig. 13 for site $(3,3)$ for zero-field SC and vortex states. The spin and site indices have been omitted.

\begin{tabular}{lcc}
\hline \hline & Zero-field SC state & Vortex state \\
\hline$\Delta_{x z}\left(\sigma_{x}\right)$ & $(0.43,0.43)$ & $(-0.12,0.58)$ \\
$\Delta_{x z}\left(\pi_{y}\right)$ & $(0.032,0.032)$ & $(-0.34,0.11)$ \\
$\Delta_{y z}\left(\pi_{x}\right)$ & $(-0.032,-0.032)$ & $(-0.12,0.34)$ \\
$\Delta_{y z}\left(\sigma_{y}\right)$ & $(-0.43,-0.43)$ & $(-0.58,0.11)$ \\
$\Delta_{x y}(\hat{x})$ & $(0.17,0.17)$ & $(0.091,0.24)$ \\
$\Delta_{x y}(\hat{y})$ & $(-0.17,-0.17)$ & $(-0.24,0.096)$ \\
\hline \hline
\end{tabular}

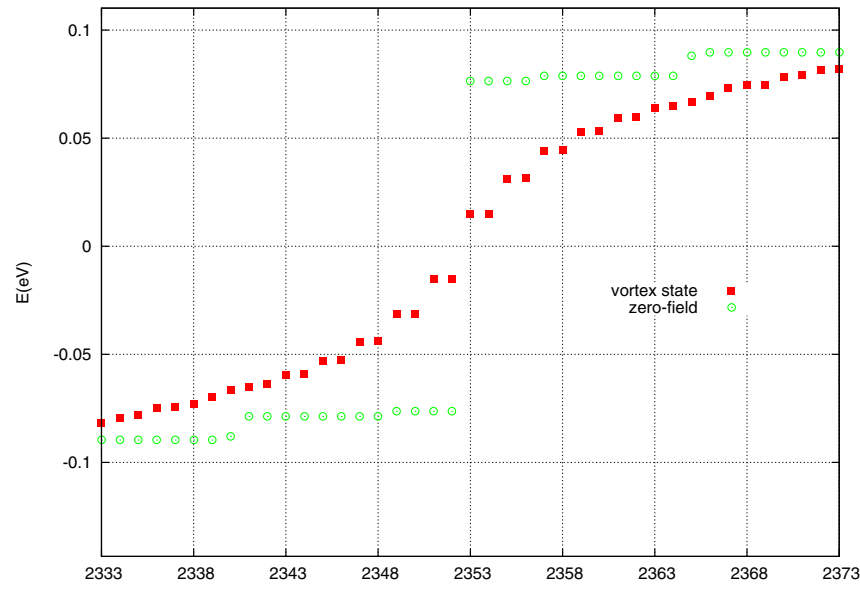

FIG. 14. (Color online) Eigenvalues of BdG equation at around the Fermi level in the cases of a $d_{x^{2}-y^{2}}$-wave pairing state for zero-field states, shown in green circles, and vortex states, shown in red squares, respectively.

$d_{x z / y z}$ orbitals and loss on the $d_{x y}$ orbital have been observed as shown in Figs. 17(e) and 17(f), which indicates a signature of charged vortex core states. However, no particle density oscillation appears in the LDOS spectrum. The superposition of in-gap bound states at site $(14,14)$ in Fig. 16(c) contributed from different orbitals reproduces a peak at the center of a vortex, which resembles the results of STM observation as shown in Fig. 16(d) [11]. The fact that the oscillation of LDOS in the case of anisotropic $s$-wave vortices is not observed in the STM measurement and the bound sates of isotropic $s$-wave vortices are extended makes us conclude that the vortex structures observed by STM may be of a $d_{x^{2}-y^{2}}$-wave feature.

We have noted that the self-consistent calculation gives different winding structures of vortex states with respect to different pairing symmetries. However, isotropic $s$ and anisotropic $s$-wave vortices share a common winding structure, which is characterized by a sink-type core state. But in the case of $d_{x^{2}-y^{2}}$-wave pairing, vortices contributed from $d_{x z / y z}$ orbitals show a phase distribution as a solenoidal vector field, whereas the $d_{x y}$ orbital shows sink- and source-type winding structures. Topologically, all these vortices correspond to a homotopy group $\pi_{1}\left[U(1), x_{0}\right]=\mathbb{Z}$. As shown in Table I, the orbital-resolved $s$ and anisotropic $s$-wave vortices belong to the same symmetry group $G_{5}$ [32], and the $d_{x^{2}-y^{2}}$-wave pairing symmetry has $d_{x z / y z}$ orbital vortices belonging to the $G_{6}^{*}$ group and $d_{x y}$ orbital vortices belonging to the $G_{5}^{*}$ group. Such results reveal that the local surgery, i.e., the continuous transformation between elements within the same homotopic class, is actually carried out by a gauge transformation, or equivalently the co-representation transformation between $G_{5}^{*}$ and $G_{6}^{*}$ [32]. The pairing bonds of orbital-resolved $d_{x^{2}-y^{2}}$-wave vortices defined on each orbital have a phase difference that is smaller than $\pi$ in the vicinity of the vortex core. Far away from the vortex core, it approaches to $\pi$ as the usual $d_{x^{2}-y^{2} \text {-wave }}$ pairing states in the case of zero magnetic field [46]. We have noted that, mathematically, the same reference point $i_{0}$ in real space can be mapped to different reference points $x_{0}$ and $x_{1}$ in $\mathrm{U}(1) \mathrm{SC}$ order parameter space, which manifest themselves as different absolute phase values, while the homotopic classes 


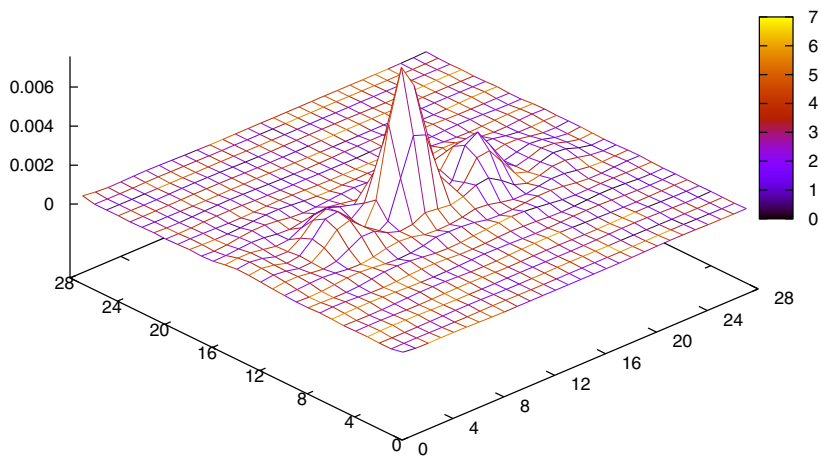

(a)

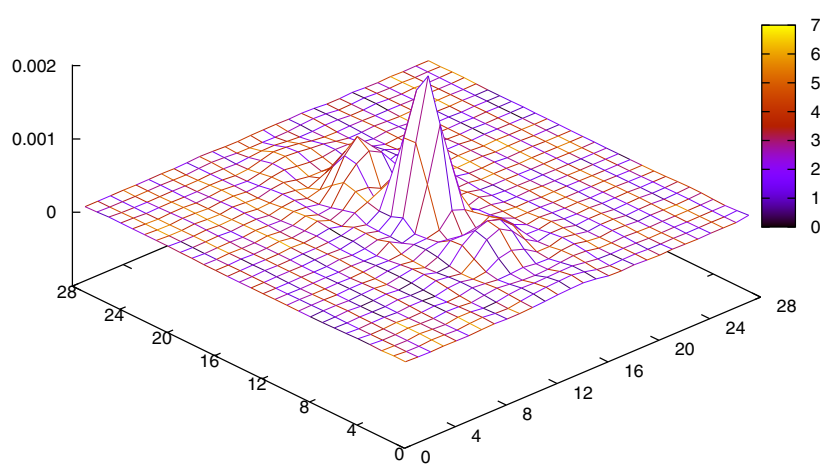

(c)

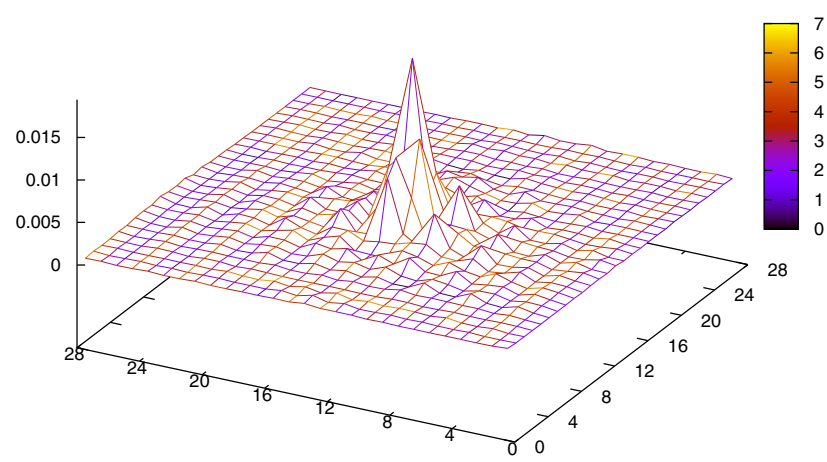

(e)

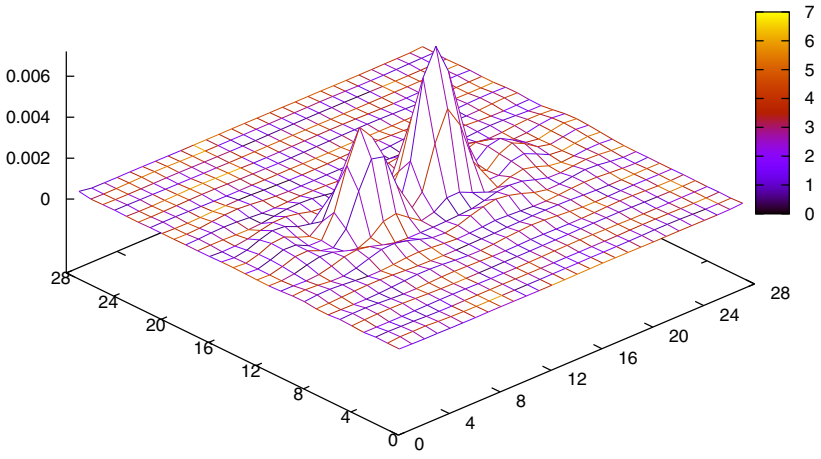

(b)

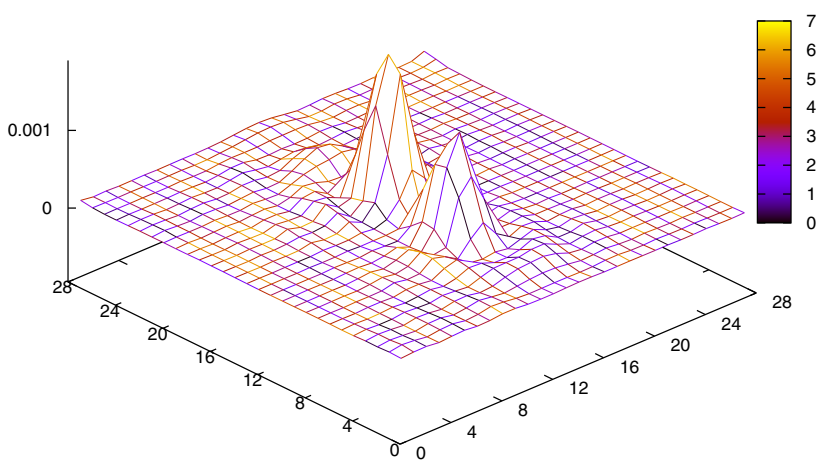

(d)

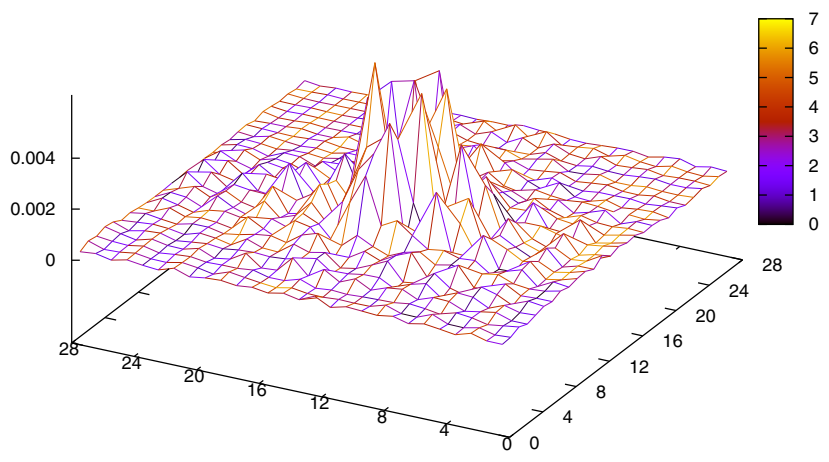

(f)

FIG. 15. (Color online) Amplitudes and phases (color mapping) of quasiparticle wave functions $u_{i \alpha \uparrow \uparrow}^{n}$ and $v_{i \alpha \downarrow \uparrow}^{n}$ for a $d_{x^{2}-y^{2}}$-wave pairing state of index $n=2353$ for a $d_{x z}$ orbital [(a) and (b)], a $d_{y z}$ orbital [(c) and (d)], and index $n=2354$ for a $d_{x y}$ orbital [(e) and (f)], respectively.

generated by $x_{0}$ and $x_{1}$ correspond to the same homotopy group $\pi_{1}[U(1), \forall x]=\mathbb{Z}$. This is why $G_{5}\left(G_{5}^{*}\right)$ and $G_{6}\left(G_{6}^{*}\right)$ subgroups have a local relative phase difference. In our numerical calculations, vortices in the case of an anisotropic $s$ wave pairing state, as shown in Fig. 18(a), show the same $G_{5}$ symmetry for different orbitals, which results in a trivial phase difference. Minor phase differences appear in the center region of the magnetic unit cell due to the amplification of the lengths of the arrows when we plot the figure. From group theoretical derivation, there is a phase difference of $\pi / 4$ between $G_{6}^{*}$ symmetry, defined on $d_{x z, y z}$ orbitals, and $G_{5}^{*}$ symmetry, defined on the $d_{x y}$ orbital, respectively, in the case of a $d_{x^{2}-y^{2}}$-wave pairing state. We have observed such a fixed phase difference from our numerical calculations as shown in Fig. 18(b). The observed phase difference is smaller than $\pi / 4$ due to the spatial anisotropy of $t_{2 g}$ orbital wave functions. As a stable topological defect, one remarkable phenomenon is that the fixed relative phase difference is essentially a signature of all the order parameters defined on the entire magnetic unit cell, which is in reality a feature originated from the topological property of U(1) gauge field. Physically, even though we have only included the intraorbital pairing, the interorbital hoppings between $d_{x z / y z}$ and $d_{x y}$ orbitals are responsible for this phase lock-in phenomenon. From a viewpoint of quasiparticle interference, the orbital degree of freedom actually gives rise to an orbital-resolved interfered phase distribution. Without loss of generality, we propose that such a phase difference between $d_{x z / y z}$ and $d_{x y}$ orbital 


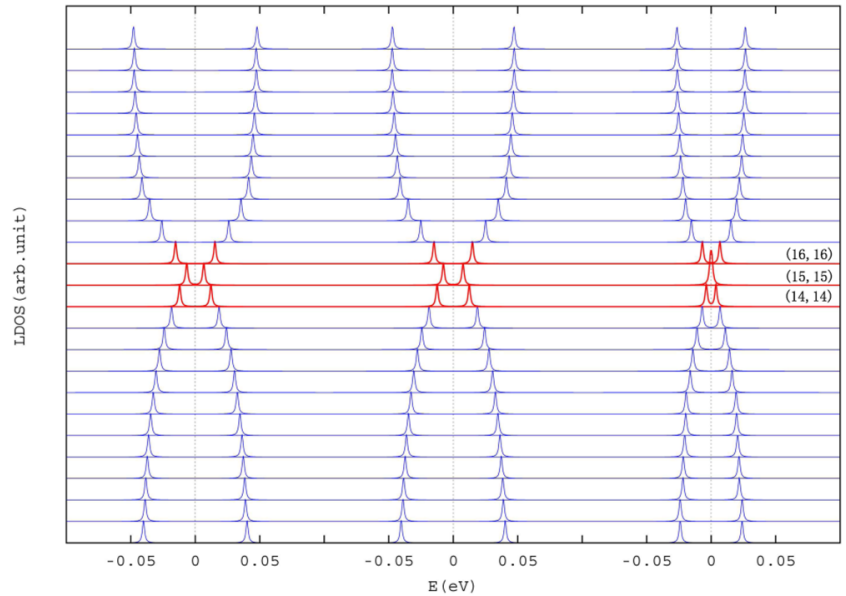

(a)

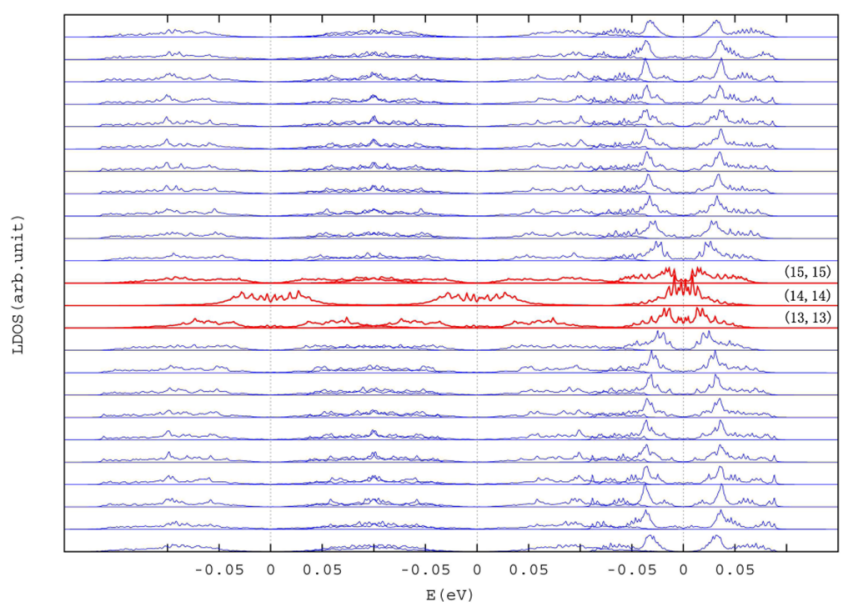

(c)

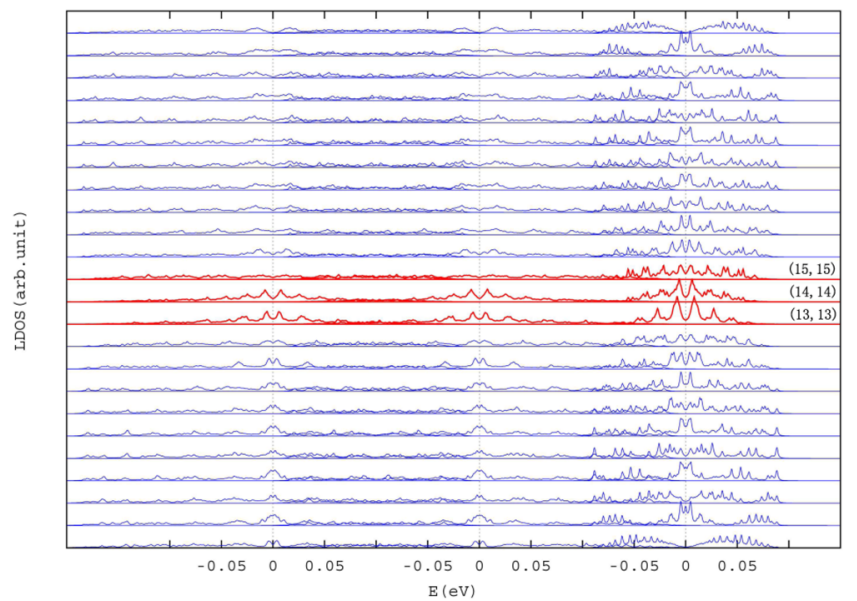

(b)

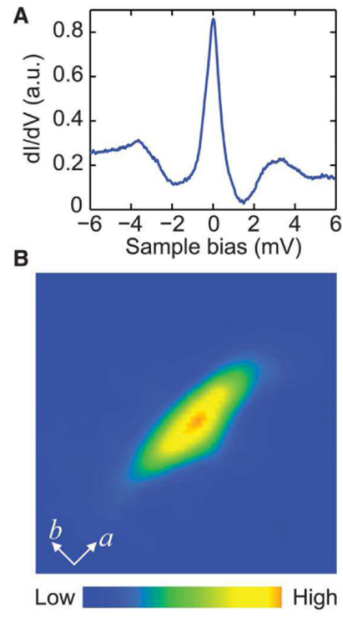

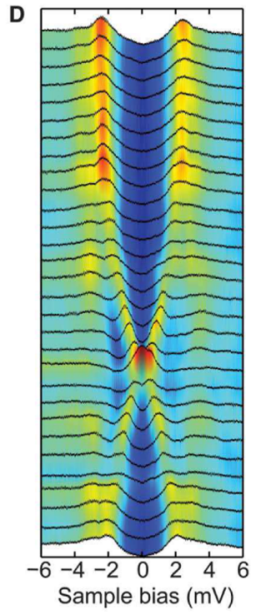

Sample bias (mV)

(d)

FIG. 16. (Color online) Orbital-resolved LDOS along an off-diagonal line from site $(3,3) \rightarrow(26,26)$. Each subfigure from left to right is

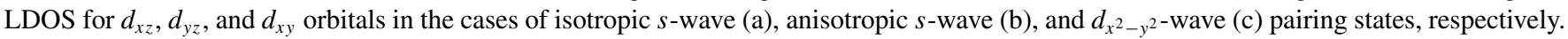
The Fermi level has been set to zero and sites in vortex region have been highlighted in red. The vortex core states from scanning tunneling spectroscopy (STS) (d). STS on the center of a vortex core A. Zero-bias conductance map for a single vortex at $0.4 \mathrm{~K}$ and 1 -T magnetic field B. Tunneling conductance curves measured at equally spaced $(2 \mathrm{~nm})$ distances along $\hat{a}$ axis $\mathbf{C}$ and $\hat{b}$ axis $\mathbf{D}$. Reprinted figure with permission from Song et al., Science 332, 1410 (2011) [11]. Copyright 2011 by American Association for the Advancement of Science (AAAS).

vortices can in principle be observed experimentally, which is independent of the specific gauge choice and, consequently, a physical manifestation of $d_{x^{2}-y^{2}}$-wave pairing states. We have confirmed that $d_{x z / y z}$ vortices always have $G_{6}^{*}$ symmetry even if we carry out an artificial gauge transformation where the relative phase of $d_{x z / y z}$ and $d_{x y}$ orbital hoppings in the band structure are changed as

$$
t_{\sigma \sigma}(i \alpha, j \beta) \rightarrow t_{\sigma \sigma}(i \alpha, j \beta) e^{i \theta_{\alpha \beta}}
$$

where $\theta_{\alpha \beta}$ is set to $\frac{\pi}{4}$ or $-\frac{3 \pi}{4}$, which is consistent with the co-representation transformation [32]. The resultant winding pattern of $d_{x z / y z}$ orbital vortices remains unchanged, while the $d_{x y}$ orbital vortex obviously changes. It turns out that if we set an equal on-site atomic energy, such phase difference of $\frac{\pi}{4}$ disappears.

\section{SUMMARY}

In summary, using a three-orbital model, we present a comprehensive investigation of single vortex core states in FeSe superconductors by means of BdG theory. The numerical results have been classified by invariant subgroups of the magnetic translation group. It turns out that isotropic $s$ - and anisotropic $s$-wave pairing symmetries give rise to $G_{5}$ vortex states. $G_{6}^{*}$ vortex states are obtained for $d_{x z / y z}$ orbitals due to orbital hybridization, and $G_{5}^{*}$ vortex states for the $d_{x y}$ orbital in the case of $d_{x^{2}-y^{2}}$-wave pairing. By analyzing the behavior of orbital-resolved quasiparticle wave functions and LDOS, and by comparing the results with STM observations, we propose that $d_{x^{2}-y^{2}}$-wave vortices are the most likely candidate. The phase difference of $\frac{\pi}{4}$ in terms of winding structures between hybridized $d_{x z / y z}$ orbitals and the $d_{x y}$ orbital can also be testified experimentally as a signature of $d_{x^{2}-y^{2}}$-wave pairing symmetry in FeSe superconductors. 


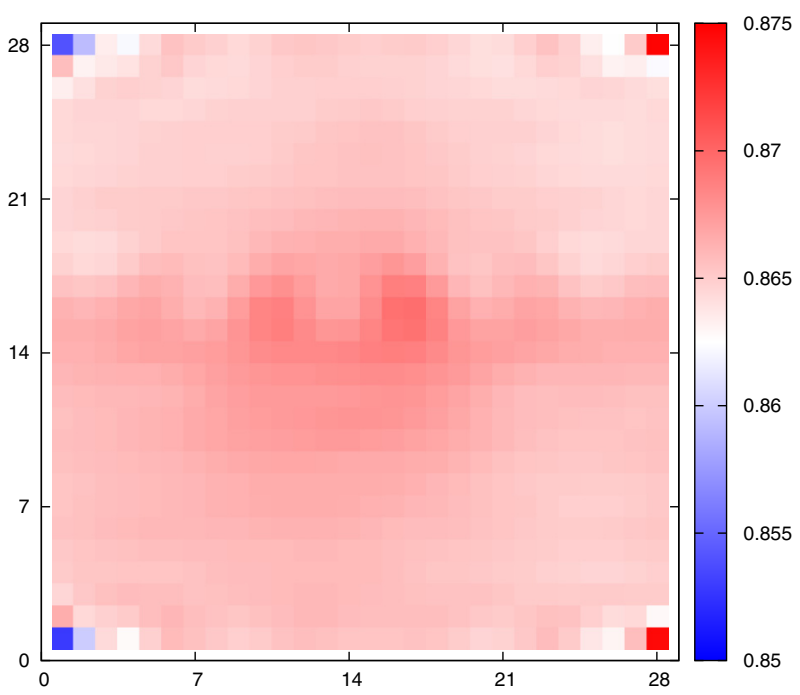

(a)

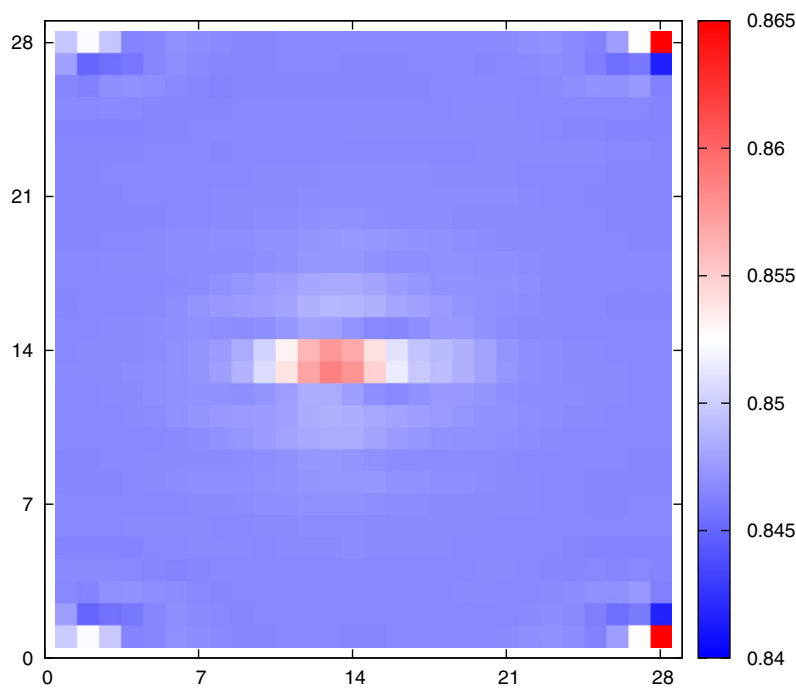

(c)

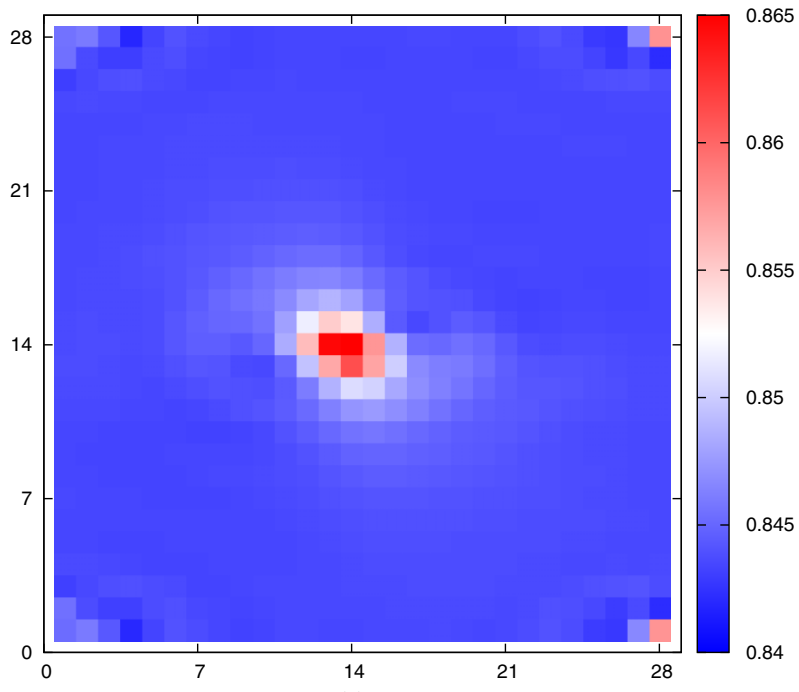

(e)

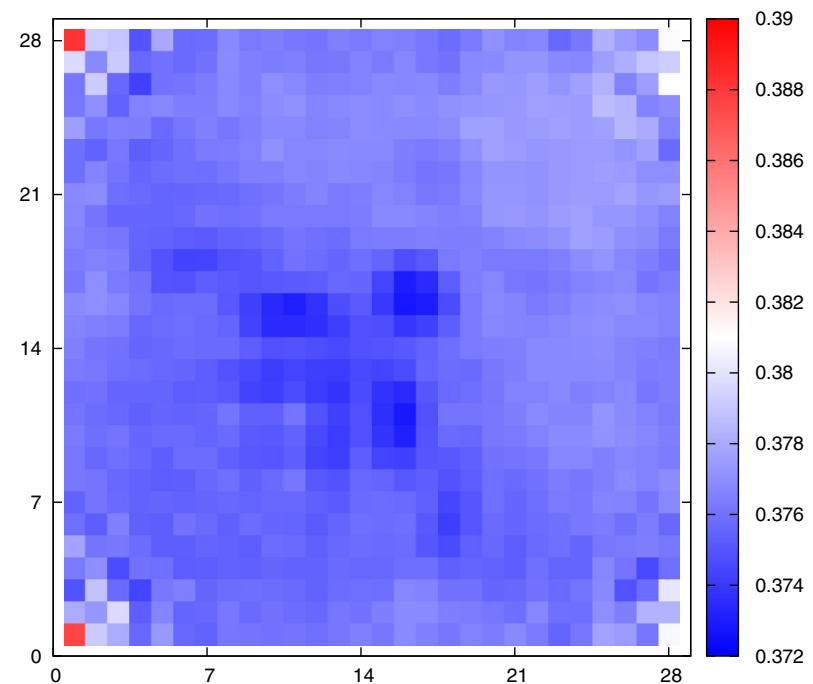

(b)

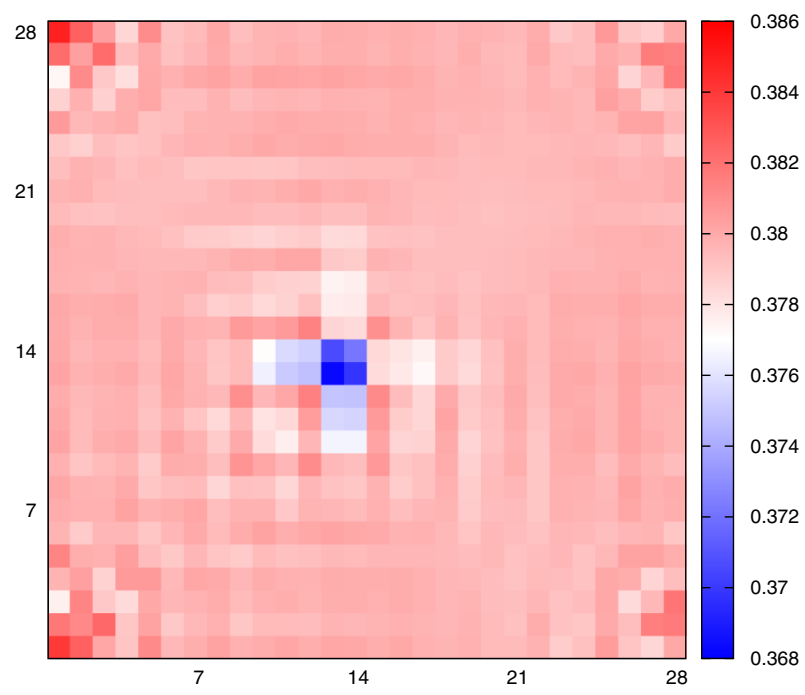

(d)

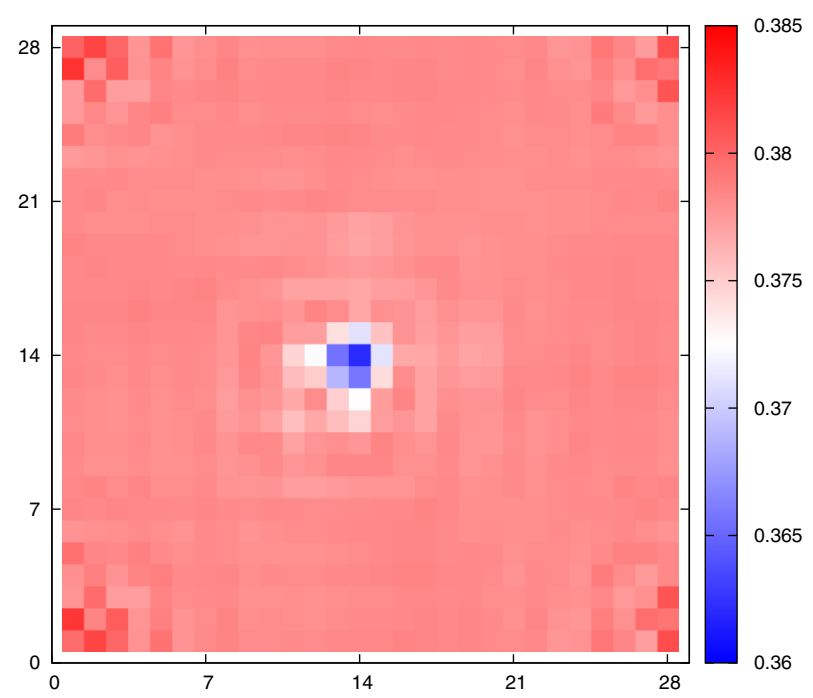

(f)

FIG. 17. (Color online) Orbital-resolved electron density for $d_{x z}$ and $d_{x y}$ orbitals for $s$-wave [(a) and (b)], anisotropic $s$ wave [(c) and (d)], and $d_{x^{2}-y^{2}}$-wave [(e) and (f)] vortices, respectively. The electron density for a $d_{y z}$ orbital in the cases of different pairing symmetries is same as for a $d_{x z}$ orbital. 


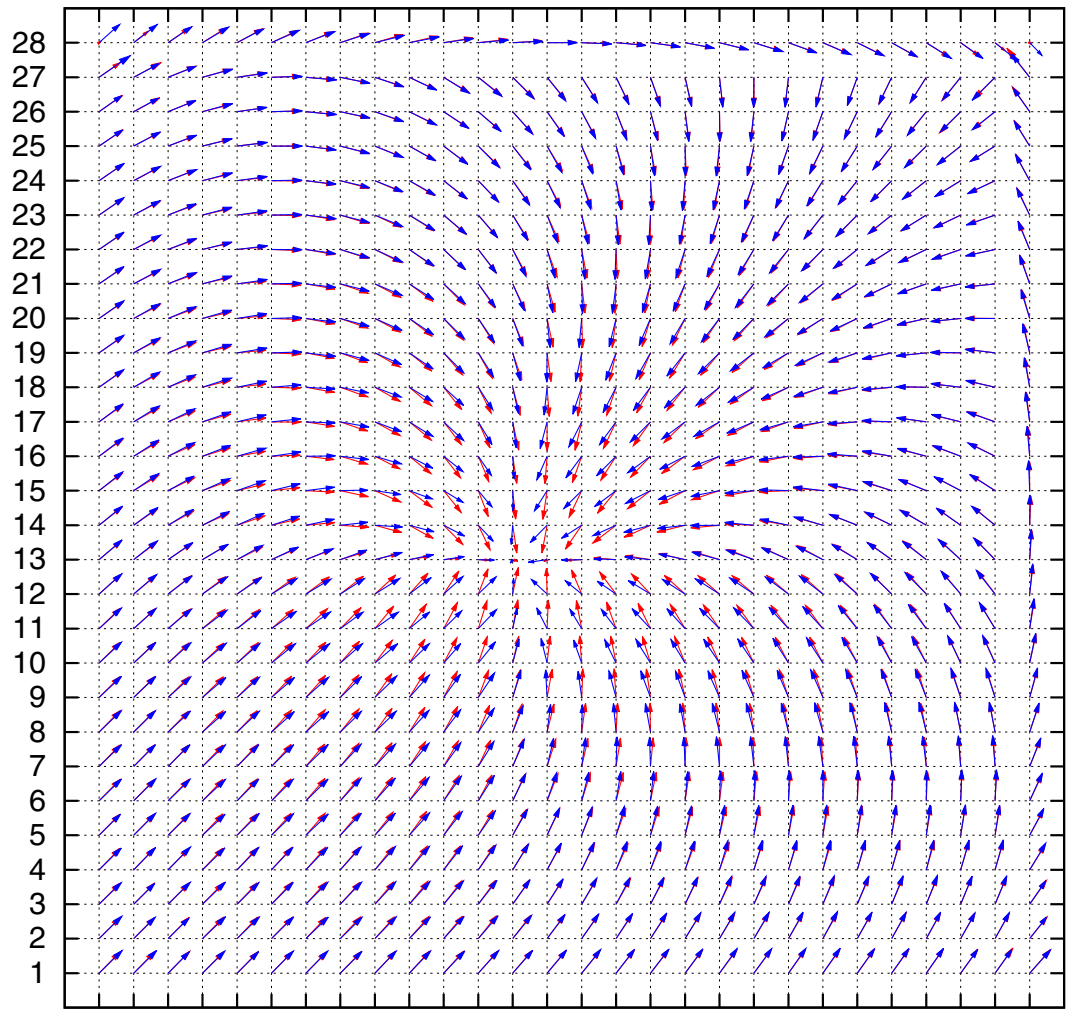

12345678910111213141516171819202122232425262728

(a)

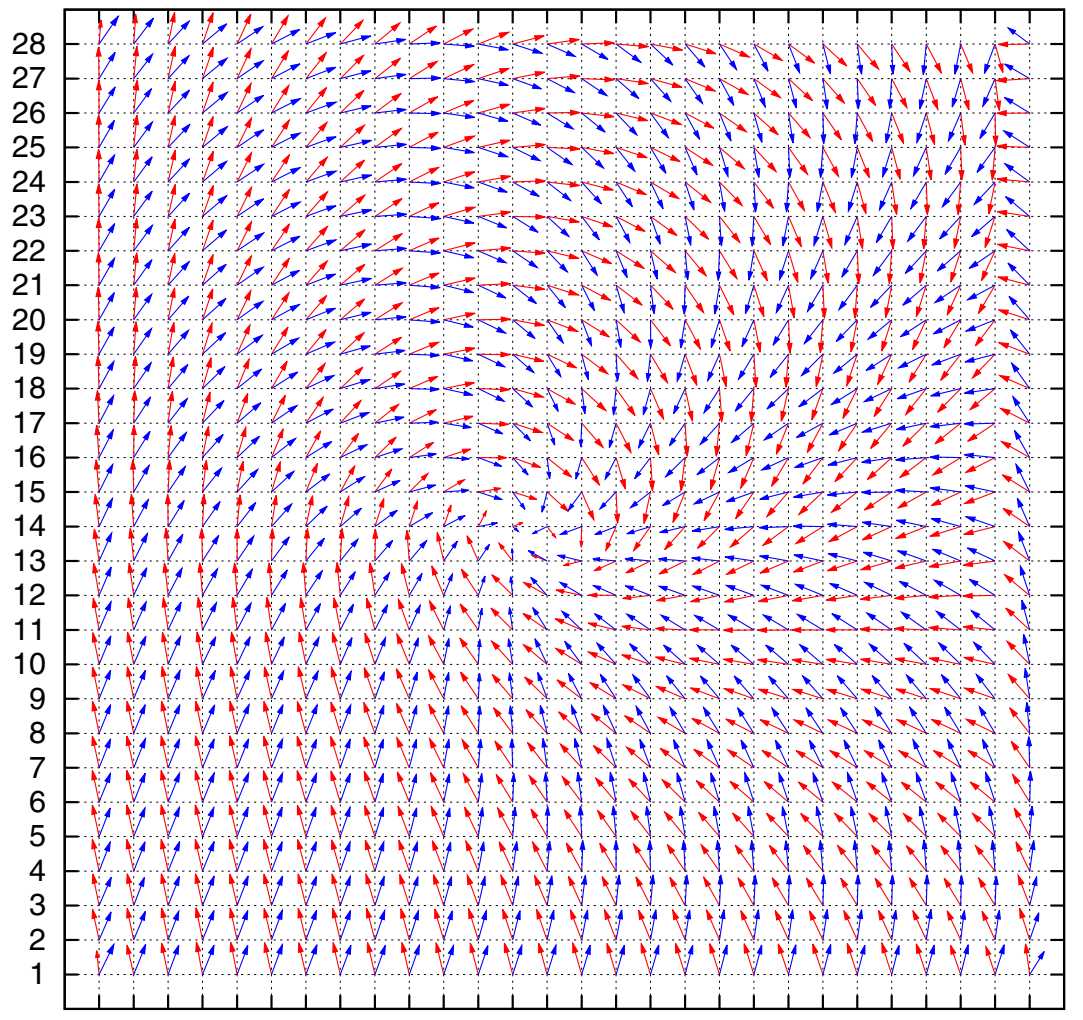

12345678910111213141516171819202122232425262728

(b)
FIG. 18. (Color online) Phase difference between $d_{x z}$ (red arrows) and $d_{x y}$ (blue arrows) orbital-resolved vortices for anisotropic $s$-wave (a) and $d_{x^{2}-y^{2}}$-wave (b) pairing states. We note that the $d_{y z}$ orbital vortices show the same symmetry as $d_{x z}$ does. This phase difference is trivial in (a) due to the same $G_{5}$ symmetry and is smaller than $\pi / 4$ from our numerical results due to the spatial anisotropy of $\mathrm{t}_{2 g}$ orbitals in (b). The arrows are amplified from the original data to obtain enough resolution. 


\section{ACKNOWLEDGMENTS}

We thank Y. Chen, Z. J. Yao, H. L. Pang, and Z. Z. Yu for inspiring discussions. We acknowledge financial support from
The Research Grant Council, University Grant Committee, Hong Kong via GRF Grant No. 706809, National Basic Research Program of China, No. 2014CB921203, and National Science Foundation of China, Grant No. 11274269.
[1] N. D. Mermin, Rev. Mod. Phys. 51, 591 (1979).

[2] D. I. Khomskii and A. Freimuth, Phys. Rev. Lett. 75, 1384 (1995).

[3] Y. Chen, Z. D. Wang, J. X. Zhu, and C. S. Ting, Phys. Rev. Lett. 89, 217001 (2002).

[4] T. Nagaoka, Y. Matsuda, H. Obara, A. Sawa, T. Terashima, I. Chong, M. Takano, and M. Suzuki, Phys. Rev. Lett. 80, 3594 (1998).

[5] G. Blatter, M. V. Feigel'man, V. B. Geshkenbein, A. I. Larkin, and V. M. Vinokur, Rev. Mod. Phys. 66, 1125 (1994).

[6] F. Gygi and M. Schlüter, Phys. Rev. B 43, 7609 (1991).

[7] Y. D. Zhu, F. C. Zhang, and M. Sigrist, Phys. Rev. B 51, 1105 (1995).

[8] Y. Wang and A. H. MacDonald, Phys. Rev. B 52, R3876 (1995).

[9] M. Takigawa, M. Ichioka, and K. Machida, J. Phys. Soc. Jpn. 69, 3943 (2000).

[10] F. C. Hsu, J. Y. Luo, K. W. Yeh, T. K. Chen, T. W. Huang, P. M. Wu, Y. C. Lee, Y. L. Huang, Y. Y. Chu, D. C. Yan, and M. K. Wu, Proc. Natl. Acad. Sci. USA 105, 14262 (2008).

[11] C. L. Song, Y. L. Wang, P. Cheng, Y. P. Jiang, W. Li, T. Zhang, Z. Li, K. He, L. Wang, J. Jia, H. H. Hung, C. Wu, X. Ma, X. Chen, and Q. K. Xue, Science 332, 1410 (2011).

[12] D. Liu, W. Zhang, D. Mou, J. He, Y. Ou, Q. Wang, Z. Li, L. Wang, L. Zhao, S. He, Y. Peng, X. Liu, C. Chen, L. Yu, G. Liu, X. Dong, J. Zhang, C. Chen, Z. Xu, J. Hu, X. Chen, X. Ma, Q. Xue, and X. J. Zhou, Nat. Commun. 3, 931 (2012).

[13] Kazuhiko Kuroki, Seiichiro Onari, Ryotaro Arita, Hidetomo Usui, Yukio Tanaka, Hiroshi Kontani, and Hideo Aoki, Phys. Rev. Lett. 101, 087004 (2008).

[14] I. I. Mazin, D. J. Singh, M. D. Johannes, and M. H. Du, Phys. Rev. Lett. 101, 057003 (2008).

[15] P. J. Hirschfeld, M. M. Korshunov, and I. I. Mazin, Rep. Prog. Phys. 74, 124508 (2011).

[16] A. Chubukov, Annu. Rev. Condens. Matter Phys. 3, 57 (2012).

[17] Fengjie Ma, Zhong-Yi Lu, and Tao Xiang, Front. Phys. China 5, 150 (2010).

[18] Qimiao Si and Elihu Abrahams, Phys. Rev. Lett. 101, 076401 (2008).

[19] Wei-Qiang Chen, Kai-Yu Yang, Yi Zhou, and Fu-Chun Zhang, Phys. Rev. Lett. 102, 047006 (2009).

[20] Kangjun Seo, B. A. Bernevig, and Jiangping Hu, Phys. Rev. Lett. 101, 206404 (2008).

[21] Fa Wang, Hui Zhai, and Dung-Hai Lee, Phys. Rev. B 81, 184512 (2010).
[22] Xianhui Chen, Pengcheng Dai, Donglai Feng, Tao Xiang, and Fu-Chun Zhang, Natl. Sci. Rev. 1, 371 (2014).

[23] R. Yu, P. Goswami, Q. Si, P. Nikolic, and J.-X. Zhu, Nat. Commun. 4, 2783 (2013).

[24] Yi Zhou, D. H. Xu, F. C. Zhang, and W. Q. Chen, Europhys. Lett. 95, 17003 (2011).

[25] X. Hu, C. S. Ting, and J. X. Zhu, Phys. Rev. B 80, 014523 (2009).

[26] T. Zhou, Z. D. Wang, Y. Gao, and C. S. Ting, Phys. Rev. B 84, 174524 (2011).

[27] H. H. Hung, C. L. Song, X. Chen, X. Ma, Q. K. Xue, and C. Wu, Phys. Rev. B 85, 104510 (2012).

[28] D. Wang, J. Xu, Y. Y. Xiang, and Q. H. Wang, Phys. Rev. B 82, 184519 (2010)

[29] M. A. N. Araújo, M. Cardoso, and P. D. Sacramento, New J. Phys. 11, 113008 (2009).

[30] A. A. Abrikosov, Sov. Phys. JETP 5, 1174 (1957).

[31] E. Brown, Phys. Rev. 133, A1038 (1964).

[32] M. Ozaki, M. Yamazaki, A. Goto, and Y. Hori, Prog. Theor. Phys. 100, 253 (1998)

[33] M. Ozaki, Y. Hori, and A. Goto, Prog. Theor. Phys. 101, 769 (1999).

[34] M. M. Salomaa and G. E. Volovik, Rev. Mod. Phys. 59, 533 (1987).

[35] R. E. Peierls, Z. Phys. 80, 763 (1933)

[36] M. Sigrist and K. Ueda, Rev. Mod. Phys. 63, 239 (1991).

[37] G. E. Volovik and L. P. Gor'kov, Sov. Phys. JETP 61, 843 (1985)

[38] I. R. Shein and A. L. Ivanovskii, Phys. Lett. A 375, 1028 (2011).

[39] C. Fang, Y.-L. Wu, R. Thomale, B. A. Bernevig, and J. Hu, Phys. Rev. X 1, 011009 (2011).

[40] F. Wang, F. Yang, M. Gao, Z. Y. Lu, T. Xiang, and D. H. Lee, Europhys. Lett. 93, 57003 (2011).

[41] N. N. Bogoljubov, V. V. Tolmachev, and D. V. Shirkov, Fortshr. Phys. 6, 605 (1958).

[42] J. G. Valatin, Nuovo Cimento 7, 843 (1958).

[43] J. X. Zhu, B. Friedman, and C. S. Ting, Phys. Rev. B 59, 3353 (1999).

[44] W. Li, H. Ding, P. Deng, K. Chang, C. Song, K. He, L. Wang, X. Ma, J. P. Hu, X. Chen, and Q. K. Xue, Nat. Phys. 8, 126 (2011).

[45] M. Daghofer, A. Nicholson, A. Moreo, and E. Dagotto, Phys. Rev. B 81, 014511 (2010).

[46] P. I. Soininen, C. Kallin, and A. J. Berlinsky, Phys. Rev. B 50, 13883(R) (1994) 